\title{
Effects of body forces on vorticity and enstrophy evolutions in turbulent premixed flames
}

Arun Ravi Varma ${ }^{1}$, Umair Ahmed ${ }^{1}$ and Nilanjan Chakraborty, ${ }^{1, *}$

${ }^{1}$ School of Engineering, Newcastle University

Claremont Road, Newcastle Upon-Tyne, NE1 7RU, UK

Email: nilanjan.chakraborty@ncl.ac.uk

\footnotetext{
* Corresponding author
} 


\section{ABSTRACT}

The effects of body forces (alternatively Froude number) on both vorticity and enstrophy evolutions within the flame brush have been analysed using Direct Numerical Simulations (DNS) data of freely propagating statistically planar turbulent premixed flames subjected to different turbulence intensities. The turbulence parameters are taken to represent the thin reaction zone regime of premixed turbulent combustion. The enstrophy has been found to decay significantly from the unburned to the burned gas side of the flame brush for high turbulence intensities and this trend is particularly prominent for the strengthening of the body force promoting unstable stratification. However, local instances of enstrophy generation have been observed and in some cases the decay of enstrophy is arrested across the flame brush for small turbulence intensities. This trend strengthens with the increasing magnitude of the body force promoting stable stratification. The enstrophy generation due to the baroclinic torque is primarily responsible for this local enstrophy generation for small turbulence intensities especially under the body force promoting stable stratification. This baroclinic torque contribution is also found to be responsible for anisotropic behaviour of vorticity components within the flame brush. The vortex stretching and viscous dissipation terms have been found to be the leading order source and sink terms, respectively, in the enstrophy transport for high turbulence intensities especially in the case of body force promoting unstable stratification. However, baroclinic torque, and the sink term due to dilatation rate continue to play significant roles even for high turbulence intensity cases considered here but their relative importance increases with decreasing turbulence intensity especially under the body force promoting stable stratification. The surface-weighted entrainment velocity has been found to be mostly unaffected by the body force in this analysis, and a minor influence can be discerned in the case of small turbulence intensities where an unstable stratification tends to promote high negative values of entrainment velocity only towards the unburned gas side of the flame brush.

Keywords: Vorticity, Enstrophy, Premixed flame, Lewis number, Direct Numerical Simulations 


\section{INTRODUCTION}

The vorticity and enstrophy fields are fundamentally important for the analysis of turbulent fluid motion $^{1-3}$ and they play key roles in determining fluid flow topology. ${ }^{4,5} \operatorname{Corrsin}^{6}$ in his pioneering work suggested a superlayer separating the vortical turbulent zone from the irrotational flow region. Libby ${ }^{7}$ characterised the interface between the vortical zone and the irrotational flow region in terms of intermittency function. Dopazo and O'Brien ${ }^{8}$ derived the exact form of the intermittency function in terms of entrainment velocity which is dependent on the enstrophy evolution in turbulent flows. However, the enstrophy evolution in premixed flames has received limited attention ${ }^{9-15}$ in comparison to the vast body of literature for turbulent non-reacting flows. ${ }^{1-8}$ The velocity pattern induced by the incompressible vortical motion gives rise to a pressure distribution which increases from the centre to the periphery of the vortex, whereas flame normal acceleration due to thermal expansion in a freely propagating laminar premixed flame without any external body force/pressure gradient gives rise to a self-induced pressure drop within the flame but the pressure eventually increases to the thermodynamic pressure in the burned gas for low Mach number combustion. ${ }^{16-25}$ The pressure gradients induced by both vortical turbulent motion and flame-normal acceleration are expected to affect the enstrophy evolution and distribution of vorticity in turbulent premixed flames through the baroclinic torque. ${ }^{9-15}$ The alignment of vorticity with local principal strain rate directions has been analysed for nonreacting ${ }^{26-32}$ flows and also for non-premixed ${ }^{33-35}$ and premixed ${ }^{9,10,14,36}$ flames. It has been found that vorticity aligns preferentially with the intermediate strain rate eigendirection but its alignments with the most extensive and the most compressive principal strain rate directions change with the regime of combustion and the characteristic Lewis number. ${ }^{7}$ It has been found that vorticity shows considerable alignment with the most compressive principal strain rate direction and no alignment with the most extensive principal strain rate direction when the dilatation rate remains almost equal to the most extensive principal strain rate, which is often realised in the corrugated flamelets regime ${ }^{37}$ and for flames with characteristic Lewis number much smaller than unity (i.e., Le « 1.0). ${ }^{10}$ However, vorticity shows an increased alignment with the most extensive principal strain rate direction in the regions of heat release for the thin reaction zones regime ${ }^{37}$ premixed turbulent flames with characteristic Lewis number close to unity (i.e., $L e \approx 1.0$ ). ${ }^{10}$ The enstrophy evolution across the flame is also determined by the 
density ratio between unburned reactants and fully burned products $\sigma$, characteristic Lewis number, and the regime of combustion. ${ }^{10,11,14}$ It has been found that enstrophy can be augmented from the unburned gas to the burned gas side of the flame for the corrugated flamelets regime $e^{37}$ and this trend is further augmented by an increase in density ratio $\sigma$ and a decrease in Le. By contrast, enstrophy drops from the unburned gas to the burned gas side for flames with high values of Karlovitz number $K a$ (e.g., for $K a \gg 1$ flames representing the thin reaction zones and broken reaction zones regimes) for $L e \approx 1.0$ but the enstrophy augmentation across the flame can also be obtained for the thin reaction zones regime flames with $L e \ll 1.0 .^{12,13}$ The evolutions of enstrophy and vorticity within the flame brush have been addressed in a number of recent analyses ${ }^{9-14}$, which revealed that $\sigma, L e$ and $\mathrm{Ka}$ have significant influence on the terms in the enstrophy transport equation. Recently, Lipatnikov et al. ${ }^{38}$ analysed the relation between enstrophy evolution and flame stretch in turbulent premixed flames in the corrugated flamelets regime and demonstrated a negative correlation between the baroclinic torque and flame stretch in these flames. However, all the aforementioned analyses on the enstrophy transport characteristics have been conducted in the absence of external pressure gradient and body forces. In contrast, premixed flames propagating in ducts are often subjected to external pressure gradients..$^{39,40}$ Moreover, the presence of buoyancy is known to affect the flame wrinkling and the nature of turbulent transport. ${ }^{39,40}$ Thus, it can be expected that the presence of external pressure gradient and body force will affect the baroclinic torque contribution to the enstrophy transport but there is no analysis available in the existing literature where the effects of external pressure gradient and body forces on the enstrophy transport are addressed. This gap in the existing literature has been addressed in the current analysis by considering a simple chemistry Direct Numerical Simulation (DNS) database of statistically planar flames subjected to different strengths of external pressure gradient/body force (i.e., characterised by Froude number $\mathrm{Fr}$, which provides the ratio of inertial force to body force) and turbulence intensities, under decaying turbulence. In this respect, the main objectives of the paper are as follows:

(a) To demonstrate the effects of $F r$ on the statistical behaviours of the different terms of the vorticity $\vec{\omega}$ and enstrophy $\Omega$ transport equations for different values of turbulence intensity. 
(b) To provide physical explanations for the influences of $F r$ and turbulence intensity on the statistical behaviours of the different terms of the vorticity $\vec{\omega}$ and enstrophy $\Omega$ transport equations.

The rest of the paper is organised as follows. The mathematical background and numerical implementation related to the current analysis are presented in Sections II and III, respectively. The results related to the influence of $F r$ on the statistical behaviours of the vorticity $\vec{\omega}$ and enstrophy $\Omega$ transport for different values of turbulence intensity are presented in Section IV. The main findings are summarised, and conclusions are drawn in Section V of this paper.

\section{MATHEMATICAL BACKGROUND}

In premixed flames, the species field is often characterised in terms of a reaction progress variable $c$, which increases monotonically from 0 in the unburned gas to 1.0 in fully burned products. The reaction progress variable $c$ can be defined based on a suitable reactant/product mass fraction $Y$ as: $c=\left(Y_{0}-\right.$ $Y) /\left(Y_{0}-Y_{\infty}\right)$ where the subscripts 0 and $\infty$ refer to the values in the unburned gas and fully burned products, respectively. The conservation equations of mass, momentum, energy and reaction progress variable take the following form in the presence of the body force term subject to the assumption of Fickian diffusion: ${ }^{39,40}$

$$
\begin{gathered}
\frac{\partial \rho}{\partial t}+\frac{\partial\left(\rho u_{j}\right)}{\partial x_{j}}=0 \\
\frac{\partial\left(\rho u_{i}\right)}{\partial t}+\frac{\partial\left(\rho u_{i} u_{j}\right)}{\partial x_{j}}=-\frac{\partial p}{\partial x_{i}}+\frac{\partial \tau_{i j}}{\partial x_{j}}+S_{i} \\
\frac{\partial(\rho e)}{\partial t}+\frac{\partial\left(\rho u_{j} e\right)}{\partial x_{j}}=-\frac{\partial\left(p u_{i}\right)}{\partial x_{i}}+\frac{\partial\left(\tau_{i j} u_{i}\right)}{\partial x_{j}}+S_{i} u_{i}+\frac{\partial}{\partial x_{j}}\left(\lambda \frac{\partial \hat{T}}{\partial x_{j}}\right)-H \frac{\partial}{\partial x_{j}}\left(\rho D \frac{\partial c}{\partial x_{j}}\right) \\
\frac{\partial(\rho c)}{\partial t}+\frac{\partial\left(\rho u_{j} c\right)}{\partial x_{j}}=\frac{\partial}{\partial x_{j}}\left(\rho D \frac{\partial c}{\partial x_{j}}\right)+\dot{\omega}_{c}
\end{gathered}
$$

where $\rho$ is the gas density, $u_{i}$ is the $i^{t h}$ component of fluid velocity, $p$ is the pressure, $\tau_{i j}$ is the component of shear stress tensor, $S_{i}=\rho \Gamma_{i}$ is the body force term in the $i^{\text {th }}$ direction, $e=\int_{T_{r e f}}^{\hat{T}} C_{v} d T^{\prime}+$ 
$u_{k} u_{k} / 2+H(1-c)$ is the specific internal energy, $\widehat{T}$ is the dimensional temperature, $C_{v}$ is the specific heat at constant volume, $\lambda$ is the thermal conductivity, $D$ is the reaction progress variable diffusivity, $H$ is heat of combustion per unit consumption of reactants and $\dot{\omega}_{c}$ is the reaction rate of reaction progress variable. The body force term is assumed to take non-zero values only in the $x_{1}$-direction, which is aligned with the mean direction of flame propagation (i.e., negative $x_{1}$-direction). Based on the body force term, a Froude number can be defined as: $F r=S_{L} / \sqrt{\Gamma_{1} \delta_{Z}}$ where $\delta_{Z}=\alpha_{T 0} / S_{L}$ is the Zel'dovich flame thickness, $S_{L}$ is the unstrained laminar burning velocity, $\alpha_{T 0}$ is the thermal diffusivity in the unburned gas. Thus, $S_{1}$ can be expressed as $S_{1}=\rho \Gamma_{1}=\rho g^{*} S_{L}^{2} / \delta_{Z}$ and $g^{*}$ stands for the inverse of Froude number-squared (i.e., $g^{*}=\Gamma_{1} \delta_{Z} / S_{L}^{2}=F r^{-2}$ ). A positive (negative) value of $g^{*}$ indicates an externally imposed flow acceleration (deceleration). Moreover, a positive (negative) value of $g^{*}$ is representative of an externally imposed adverse (favourable) pressure gradient. A positive value of $g^{*}$ also represents an unstable configuration where the heavier cold unburned reactants reside on top of the lighter hot burned products whereas a negative $g^{*}$ value is indicative of a stable configuration where the heavier cold unburned reactants reside underneath the lighter hot burned products.

One obtains the transport equation of vorticity $\vec{\omega}=\nabla \times \vec{u}$ upon taking curl of eq. $1 \mathrm{~b}$. The transport equation of the $i^{\text {th }}$ component of vorticity $\omega_{i}=\varepsilon_{i j k} \partial u_{k} / \partial x_{j}$ takes the following form: ${ }^{11-15}$

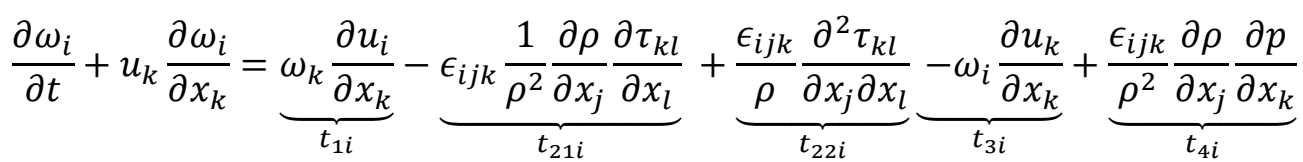

The term $t_{l i}$ on the right-hand side of eq. 2 is the $i^{\text {th }}$ component of the vortex-stretching term, whereas $t_{21 i}$ represents the $i^{\text {th }}$ component of the viscous torque term arising from the misalignment between the gradients of viscous stress and density and vanishes in constant-density flows. The $i^{\text {th }}$ component of the third term on the right-hand side (i.e., $t_{22 i}$ ) is the vorticity diffusion term and is equal to $v \partial^{2} \omega_{i} / \partial x_{j} \partial x_{j}$ in Newtonian fluid flows with constant thermo-physical properties with $v$ being the kinematic viscosity. The term $t_{3 i}$ depicts the destruction of the $i^{\text {th }}$ component of vorticity by dilatation rate, whereas the $i^{\text {th }}$ component of term $\overrightarrow{t_{4}}$ represents the contribution from the baroclinic torque owing to the misalignment 
of the density and pressure gradients. The terms $\overrightarrow{t_{21}}, \overrightarrow{t_{3}}$ and $\overrightarrow{t_{4}}$ vanish in constant-density flows. Note that the curl of $S_{i}$ is zero as the imposed body force has a constant value. Consequently, the source term $S_{i}$ does not introduce any additional term in the transport equation of $\omega_{i}$ but this source term can lead to differences in pressure gradient which can lead to differences in the behaviours of the baroclinic torque term $\overrightarrow{t_{4}}$ in response to the variations of $\mathrm{Fr}$.

On multiplication of both sides of eq. 2 by $\omega_{i}$ the transport equation of enstrophy $\Omega=\omega_{i} \omega_{i} / 2^{11-15}$ is obtained :

$$
\frac{\partial \Omega}{\partial t}+u_{k} \frac{\partial \Omega}{\partial x_{k}}=\underbrace{\omega_{i} \omega_{k} \frac{\partial u_{i}}{\partial x_{k}}}_{T_{1}} \underbrace{\epsilon_{i j k} \omega_{i} \frac{1}{\rho^{2}} \frac{\partial \rho}{\partial x_{j}} \frac{\partial \tau_{k l}}{\partial x_{l}}}_{T_{21}}+\underbrace{\frac{\epsilon_{i j k} \omega_{i}}{\rho} \frac{\partial^{2} \tau_{k l}}{\partial x_{j} \partial x_{l}}}_{T_{22}} \underbrace{-2 \frac{\partial u_{k}}{\partial x_{k}}}_{T_{3}} \Omega \underbrace{\epsilon_{i j k} \frac{\omega_{i}}{\rho^{2}} \frac{\partial \rho}{\partial x_{j}} \frac{\partial p}{\partial x_{k}}}_{T_{4}}
$$

Reynolds averaging eq. 3 yields the following equation for the Reynolds averaged enstrophy $\bar{\Omega}^{11,12,14,15}$ :

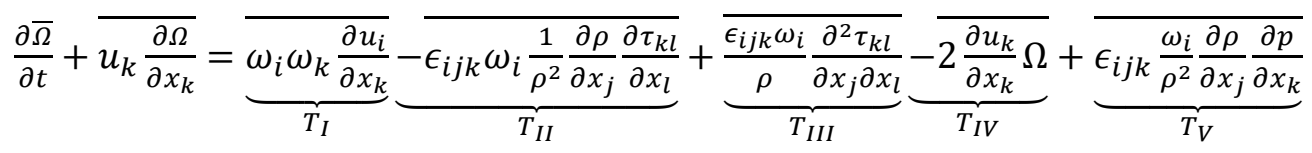

where $\bar{Q}$ indicates the Reynolds averaged value of a general quantity $Q$. The Favre average of a general quantity $Q$ is given by: $\tilde{Q}=\bar{\rho} \bar{Q} / \bar{\rho}$ and $Q^{\prime \prime}=Q-\tilde{Q}$ is the Favre fluctuation of the quantity $Q$. In the case of isotropic turbulence, the dissipation rate $\tilde{\varepsilon}$ of turbulent kinetic energy $\tilde{k}=\overline{\rho u_{\imath}^{\prime \prime} u_{\imath}^{\prime \prime}} / 2 \bar{\rho}$ can be expressed as: $\bar{\rho} \tilde{\varepsilon} \approx 2 \mu \bar{\Omega}$ under the assumption of constant viscosity and therefore the transport of $\bar{\Omega}$ is important for the purpose of the closure of dissipation rate $\tilde{\varepsilon}^{1,11}$ The term $T_{I}$ is the vortex stretching contribution to the mean enstrophy $\bar{\Omega}$ transport, whereas $T_{I I}$ represents the mean value of the crossproduct of two vectors, the density gradient and the viscosity torque. The term $T_{I I I}$, can be rewritten as: $\overline{v \omega_{l}\left(\partial^{2} \omega_{l} / \partial x_{J} \partial x_{J}\right)}=\overline{v \partial^{2} \Omega / \partial x_{J}^{2}}-\overline{v\left(\partial \omega_{l} / \partial x_{J}\right)\left(\partial \omega_{l} / \partial x_{J}\right)}$ if the dynamic viscosity is constant, and it represents the combined action of molecular diffusion (i.e., $\overline{v \partial^{2} \Omega / \partial x_{J}^{2}}$ ) and dissipation (i.e., $\left.-\overline{v\left(\partial \omega_{l} / \partial x_{J}\right)\left(\partial \omega_{l} / \partial x_{J}\right)}\right)$ of the mean enstrophy $\bar{\Omega}$. The dissipation contribution (i.e., $\left.-\overline{v\left(\partial \omega_{l} / \partial x_{J}\right)\left(\partial \omega_{l} / \partial x_{J}\right)}\right)$ dominates over the molecular diffusion term (i.e., $\overline{v \partial^{2} \Omega / \partial x_{J}^{2}}$ ) for high 
values of turbulent Reynolds number. The term $T_{I V}$ represents enstrophy dissipation due to dilatation whereas the baroclinic torque contribution to the enstrophy transport is represented by $T_{V}$. The statistical behaviours $T_{I}, T_{I I}, T_{I I I}, T_{I V}$ and $T_{V}$ in response to the variations of turbulence intensity and $g^{*}=F^{-2}$ will be discussed in detail in Section IV of this paper.

\section{NUMERICAL IMPLEMENTATION}

A well-known compressible DNS code SENGA $+{ }^{41}$ is used to generate the database in the present study. A single step irreversible chemical reaction has been considered for the purpose of simplification of chemistry in the interest of an extensive parametric analysis in terms of turbulence intensity and $g^{*}=$ $\mathrm{Fr}^{-2}$. As the present analysis focuses on the fluid-dynamical aspects of turbulent premixed combustion, the simplification in terms of chemistry is not expected to influence the results of this paper and the conclusions drawn from them. Previous studies demonstrated that the enstrophy statistics obtained from simple chemistry DNS data ${ }^{12,14,42}$ remain in good qualitative agreement with the corresponding statistics obtained from detailed chemistry DNS. ${ }^{12,39}$ In SENGA+, all the spatial derivates for the internal grid points are evaluated using a $10^{\text {th }}$ order central difference scheme and the order of accuracy gradually drops to a one-sided $2^{\text {nd }}$ order scheme at the non-periodic boundaries. ${ }^{41}$ The time advancement has been carried out using a low-storage $3^{\text {rd }}$ order Runge-Kutta scheme. ${ }^{43}$ The Lewis number of all species are considered to be unity and standard values are considered for the Prandtl number $\operatorname{Pr}(\operatorname{Pr}=0.7)$ and Zel'dovich number $\beta=T_{a c}\left(T_{a d}-T_{0}\right) / T_{a d}^{2}(\beta=6.0)$, where $T_{a c}$ is the activation temperature. The heat release parameter $\tau=\sigma-1=\left(T_{a d}-T_{0}\right) / T_{0}$ is taken to to be 4.5 (i.e., $\tau=4.5$ and $\sigma=5.5$ ). The gaseous reacting mixture is assumed to follow ideal gas law.

A schematic diagram of the simulation domain is provided in Fig. 1, and it can be seen that the boundaries in the direction of mean flame propagation (i.e., $x_{1}$-direction) are taken to be partially nonreflecting and the other boundaries are taken to be periodic. The non-periodic boundary conditions are specified using the Navier-Stokes Characteristic Boundary Conditions (NSCBC) technique. ${ }^{44} \mathrm{~A}$ divergence free, homogeneous, isotropic turbulence field generated using the pseudo-spectral method 
of Rogallo ${ }^{45}$ according to a Batchelor- Townsend kinetic energy spectrum ${ }^{46}$ is used to initialise the turbulent velocity field. The flame and reacting scalar fields have been initialised by steady-state onedimensional unstretched laminar premixed flame solution. The initial values of normalised root-meansquare turbulent velocity fluctuation $u^{\prime} / S_{L}$, integral length scale to flame thickness ratio $l_{T} / \delta_{t h}$, Damköhler number $D a=l_{T} S_{L} / u^{\prime} \delta_{t h}$ and Karlovitz number $K a=\left(u^{\prime} / S_{L}\right)^{1.5}\left(l_{T} / \delta_{t h}\right)^{-0.5}$ are presented in Table I. Based on the values of $u^{\prime} / S_{L}$ and $l_{T} / \delta_{t h}$ listed in Table I, all flames considered in this analysis belong to the thin reaction zones regime of combustion. ${ }^{34}$ In the present work the simulations have been performed for $g^{*}=F^{-2}=-3.12,-1.56,0.0,1.56,3.12$ for each set of turbulence parameters summarised in Table I. A rectangular domain of size $70.2 \delta_{\mathrm{Z}} \times 35.1 \delta_{\mathrm{Z}} \times 35.1 \delta_{\mathrm{Z}}$, which amounts to be $12 l_{\mathrm{T} 0} \times 6 l_{\mathrm{T} 0} \times 6 l_{\mathrm{T} 0}$ where $l_{\mathrm{T} 0}$ is the initial integral length scale of turbulence has been used to carry out the simulations. The integral length scale typically increased by a factor of 1.22 at the time statistics were extracted and thus each side of the simulation domain contains enough independent turbulent samples. The domain dimensions in terms of integral length scale considered for this analysis remain either comparable or bigger than the analyses which focussed on the vorticity and enstrophy evolution in turbulent premixed flames in the past. ${ }^{9,11-15}$ It has also been assessed that halving the sample size in the transverse directions does not affect both flame wrinkling and vorticity transport statistics. The simulation domain is discretised by a Cartesian mesh of $400 \times 200 \times 200$ with equidistant grid spacing, ensuring that the grid spacing $\Delta x$ remains smaller than the Kolmogorov length scale and 10 grid points are accommodated within the thermal flame thickness $\delta_{t h}=\left(T_{a d}-T_{0}\right) / \max |\nabla \widehat{T}|_{L}$. The Kolmogorov length scale can be estimated as: $\eta \sim l_{T} /\left(D a^{3 / 2} K a^{3 / 2}\right)$, which can also be obtained from the analytical expression of dissipation rate of kinetic energy for the Batchelor-Townsend spectrum. ${ }^{46}$ At least one Kolmogorov length scale is resolved by 1.2 grid points for the $u^{\prime} / S_{L}=10$ case at the initial condition and a greater number of grid points resides within the Kolmogorov length scale as turbulence decays. This implies that in the case of lower initial $u^{\prime} / S_{L}$ cases (i.e., 3.0 and 5.0) the Kolmogorov length scale is resolved by more than 1.5-2.0 grid points as the grid spacing is kept constant across all the cases investigated in this paper. As $\delta_{t h} / \eta$ scales as $\delta_{t h} / \eta \sim K a^{0.5}$, the length scale $\delta_{t h} / 10$ is smaller than $\eta$ for all cases considered here because $K a$ remains significantly smaller than 100 . Thus, the flame 
resolution determines the grid spacing for the cases considered here. It has been found that the profiles of reaction progress variable, temperature, and density do not change appreciably when the grid is made either coarser or finer by a factor of 2.0 and the magnitudes of the differences in $S_{L}$ and $\delta_{t h}$ for these grid spacings remain less than $0.1 \%$. All simulations have been carried out for $3.0 t_{e}$ (where $t_{e}=l_{T} / u^{\prime}$ is the initial eddy turnover time), which is greater than $2 \delta_{t h} / S_{L}$ for all the cases considered. The turbulent kinetic energy and dissipation rate evaluated over the unburned gas volume did not change significantly with time when the statistics are extracted. By that time, $u^{\prime}$ decayed by $55 \%$ and $l_{T}$ increased by $22 \%$ in comparison to their initial values. The simulation time remains comparable to several studies ${ }^{11,14,15,40,47-51}$ which contributed to the enstrophy and vorticity transport analysis in the past. The Reynolds/Favre-averaged values of a general quantity $q$ are evaluated by ensemble averaging the relevant quantities in $x_{2}-x_{3}$ planes at a given $x_{1}$ location following several previous analyses. $11,14,15,40,47,48$

Table I. Initial values of numerical parameters considered for the simulations

\begin{tabular}{ccccccc}
\hline & $\boldsymbol{u}^{\prime} / \boldsymbol{S}_{\boldsymbol{L}}$ & $\boldsymbol{l}_{\boldsymbol{T}} / \boldsymbol{\delta}_{\boldsymbol{t h}}$ & $\boldsymbol{D a}$ & $\boldsymbol{K a}$ & Domain size & Grid size \\
\hline Set-A & 3.0 & 3.0 & 1.0 & 3.0 & $70.2 \delta_{Z} \times\left(35.1 \delta_{Z}\right)^{2}$ & $400 \times(200)^{2}$ \\
Set-B & 5.0 & 3.0 & 0.6 & 6.45 & $70.2 \delta_{Z} \times\left(35.1 \delta_{Z}\right)^{2}$ & $400 \times(200)^{2}$ \\
Set-C & 7.5 & 3.0 & 0.4 & 11.86 & $70.2 \delta_{Z} \times\left(35.1 \delta_{Z}\right)^{2}$ & $400 \times(200)^{2}$ \\
Set-D & 10.0 & 3.0 & 0.3 & 18.26 & $70.2 \delta_{Z} \times\left(35.1 \delta_{Z}\right)^{2}$ & $400 \times(200)^{2}$ \\
\hline
\end{tabular}

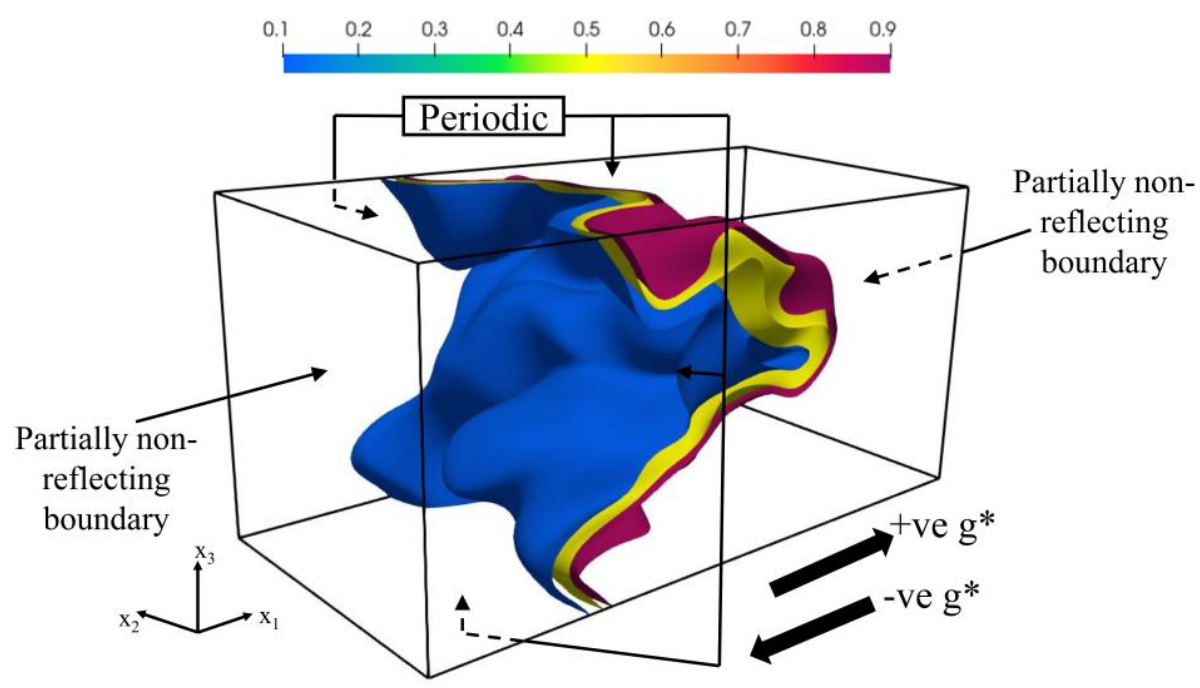

Figure 1: Schematic diagram of the simulation domain. 


\section{RESULTS \& DISCUSSION}

The distributions of normalised vorticity magnitude $\sqrt{\omega_{i} \omega_{i}} \times \delta_{Z} / S_{L}$ at the central mid-plane for the cases considered here are shown in Fig. 2 at the time when the statistics are extracted. The contours of reaction progress variable $c=0.1$ to 0.9 (in steps of 0.1 from left to right) are also superimposed on the normalised vorticity magnitude distribution in Fig. 2. The temporal evolutions of $c=0.3,0.5,0.7$ and 0.9 isosurfaces for Set-A and Set-D with $g^{*}=-3.12$ and $g^{*}=3.12$ are exemplarily shown in Appendix A for understanding the temporal evolution of flame-turbulence interaction. It can be seen from Fig. 2 that the extent of flame wrinkling is smaller for negative $g^{*}$ values than for positive $g^{*}$ values, especially for small turbulence intensities. Moreover, for a given value of $g^{*}$, the flame wrinkling increases with increasing turbulence intensity $u^{\prime} / S_{L}$ because of greater extent of flame surface distortion. The increasing extent of flame wrinkling with increasing values of $g^{*}$ and $u^{\prime} / S_{L}$ can be substantiated from the increasing trends of normalised flame surface area $A_{T} / A_{L}$ and the normalised volume-integrated reaction rate $\mathrm{W}_{T} / \mathrm{W}_{L}$ with increases in $g^{*}$ and $u^{\prime} / S_{L}$, as observed from Fig. 3 where $A=\int_{V}|\nabla c| d V$ and $\mathrm{W}=\int_{\mathrm{V}} \dot{\omega}_{c} d V$ with $\dot{\omega}_{c}$ being the reaction rate of reaction progress variable, and subscripts $T$ and $L$ are used for turbulent and steady laminar flow conditions. A positive value of $g^{*}$ is representative of the unstable stratification where the heavier reactants are placed on top of the lighter products, which acts to augment the turbulence-induced flame wrinkling. By contrast, a negative value of $g^{*}$ is representative of the stable stratification where the lighter products sit on the heavier reactants and thus the oncoming turbulent flow-induced disturbances are opposed by stable stratification of density. It can be seen from Fig. 2, and the values of $A_{T} / A_{L}$ and $\mathrm{W}_{T} / \mathrm{W}_{L}$ in Fig. 3 that the effects of $g^{*}$ are most prominent for small values of $u^{\prime} / S_{L}$ and the body force effects weaken with increasing $u^{\prime} / S_{L}$. 


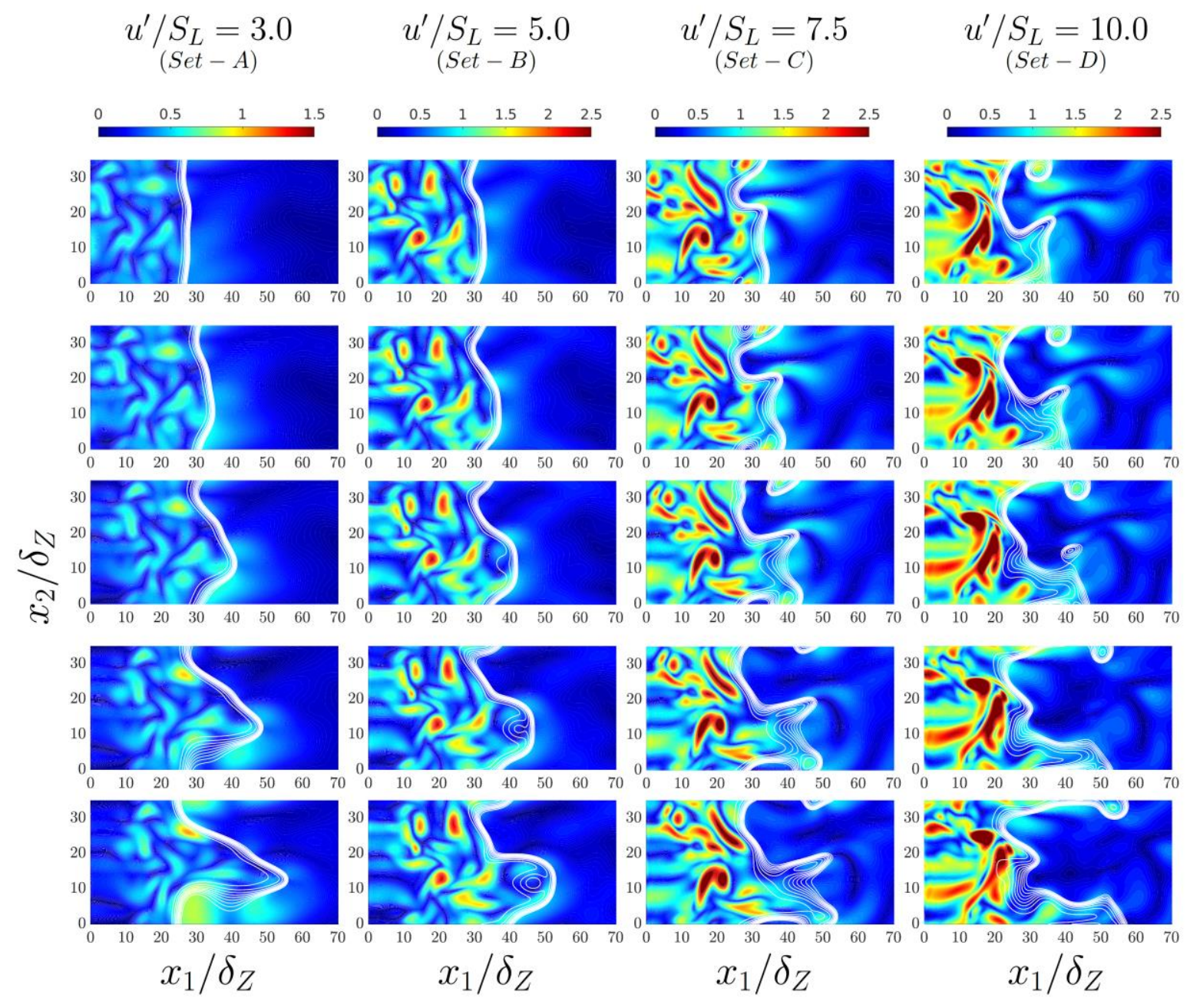

Figure 2: Distribution of $\left(\omega_{i} \omega_{i}\right)^{1 / 2} \times \delta_{Z} / S_{L}$ in the central $x_{1}-x_{2}$ plane at time when the statistics are extracted for Set-A to Set-D $\left(1^{\text {st }}-4^{\text {th }}\right.$ column $)$ for $g^{*}=-3.12,-1.56,0.0,1.56$ and $3.12\left(1^{\text {st }}-5^{\text {th }}\right.$ row). The contours of reaction progress variable $c=0.1$ to 0.9 (in steps of 0.1 from left to right) are also superimposed on the normalised vorticity magnitude distribution.

In order to understand this behaviour it is worthwhile to consider the ratio of body forces to the inertial forces due to turbulent velocity fluctuations as: $F r_{T}^{-2}=\Gamma_{1} l_{T} / u^{\prime 2}=\left(g^{*} S_{L}^{2} / \delta_{Z}\right) /\left(u^{\prime 2} / l_{T}\right) \sim g^{*}\left(l_{T} / \delta_{Z}\right) /$ $\left(u^{\prime 2} / S_{L}^{2}\right)$ where $F r_{T}=u^{\prime} / \sqrt{\Gamma_{1} l_{T}}$ is the turbulent Froude number. This suggests that for a given set of values of $l_{T} / \delta_{Z}$ and $g^{*}, F r_{T}$ decreases with increasing $u^{\prime} / S_{L}$ suggesting a diminishing effect of the body force in comparison to the inertial forces. Thus, at high values of turbulence intensity, the body force plays a marginal role and therefore the variation of $g^{*}$ does not affect both vorticity distribution and flame wrinkling for high values of $u^{\prime} / S_{L}$ (e.g., $u^{\prime} / S_{L}=7.5$ and 10.0 cases). 

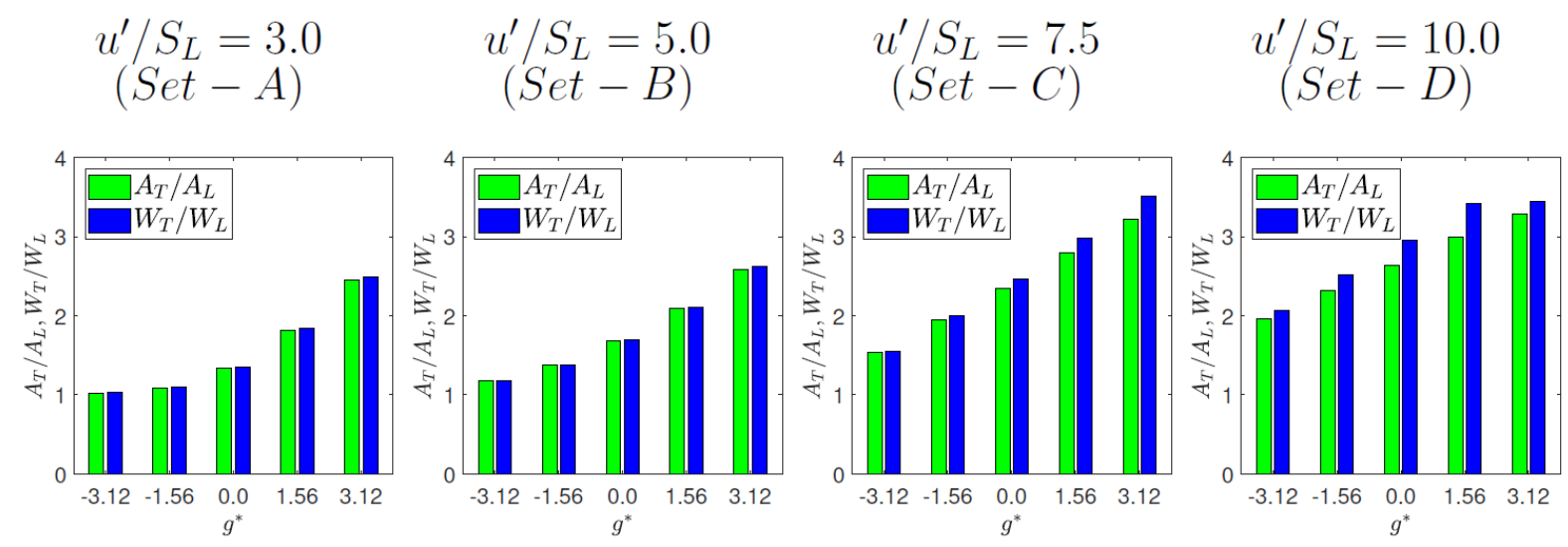

Figure 3: Variations of $A_{T} / A_{L}$ and $W_{T} / W_{L}$ with $g^{*}$ for all sets of turbulence parameters considered.

Figure 2 indicates that $\sqrt{\omega_{i} \omega_{i}} \times \delta_{Z} / S_{L}$ mostly decays from the unburned to the burned gas side of the flame for high values of $u^{\prime} / S_{L}$ (e.g., initial $u^{\prime} / S_{L}=7.5$ and 10.0 cases), whereas the instances of localised augmentation of the vorticity magnitude can be discerned across the flame for small and moderate values of turbulence intensity (e.g., initial $u^{\prime} / S_{L}=3.0$ and 5.0 cases) irrespective of the value of $g^{*}$. This localised vorticity generation across the flame is not only evident for initial $u^{\prime} / S_{L}=3.0$ and 5.0 cases but also can be seen for initial $u^{\prime} / S_{L}=7.5$ cases albeit with a reduced strength. The effects of $g^{*}$ on the vorticity magnitude are marginal for the cases with initial $u^{\prime} / S_{L}=10.0$. This is also consistent with the expected marginal influence of body forces for large turbulence intensities as a result of the weakening of body forces relative of the inertial forces.

The variations of the Reynolds averaged normalised vorticity magnitude $\overline{\left(\omega_{l} \omega_{l}\right)^{1 / 2}} \times \delta_{Z} / S_{L}$, and its flame normal $\overline{\left(\omega_{n} \omega_{n}\right)^{1 / 2}} \times \delta_{Z} / S_{L}$ (here the repeated indices do not indicate summation) and flame tangential $\overline{\left(\omega_{t} \omega_{t}\right)^{1 / 2}} \times \delta_{Z} / S_{L}$ (here the repeated indices indicate summation over two tangential directions) contributions with Favre-averaged reaction progress variable $\tilde{c}$ are shown in Fig. 4 for cases with different values of $u^{\prime} / S_{L}$ and $g^{*}$ where $\omega_{n} \omega_{n}=N_{i} N_{j} \omega_{i} \omega_{j}$ and $\omega_{t} \omega_{t}=\left(\delta_{i j}-N_{i} N_{j}\right) \omega_{i} \omega_{j}$ are the flame normal and flame tangential components of $\omega_{i} \omega_{i}$ respectively with $N_{i}=-\left(\partial c / \partial x_{i}\right) /|\nabla c|$ being the $i^{\text {th }}$ component of the local flame normal vector. As statistically planar flame cases are considered here, all Reynolds/Favre averaged values are only the function of the coordinate which is aligned with 
the mean direction of flame propagation (i.e., $x_{1}$-direction). Thus, all the results in Fig. 4 and subsequent figures are shown as a function of the Favre-averaged reaction progress variable $\tilde{c}$. It can be seen from Fig. 4 that $\overline{\left(\omega_{n} \omega_{n}\right)^{1 / 2}}$ mostly decays from the unburned to burned gas side of the flame for all cases. By contrast, $\overline{\left(\omega_{t} \omega_{t}\right)^{1 / 2}}$ decays from the unburned gas side of the flame brush before rising again for negative values of $g^{*}$ in the cases with small values of turbulence intensity (e.g., initial $u^{\prime} / S_{L}=3.0$ cases). However, this trend weakens with increasing values of $u^{\prime} / S_{L}$ and $g^{*}$ but localised augmentations of $\overline{\left(\omega_{l} \omega_{l}\right)^{1 / 2}}$ are obtained for initial $u^{\prime} / S_{L}=7.5$ and 10.0 cases for $g^{*}=-1.56$ and 3.12. Figure 4 suggests that $\overline{\left(\omega_{t} \omega_{t}\right)^{1 / 2}}$ is principally responsible for the localised augmentation of $\overline{\left(\omega_{l} \omega_{l}\right)^{1 / 2}}$ for negative values of $g^{*}$ under small values of turbulence intensity. It can further be appreciated from Fig. 4 that $\overline{\left(\omega_{t} \omega_{t} / 2\right)^{1 / 2}}$ and $\overline{\left(\omega_{n} \omega_{n}\right)^{1 / 2}}$ are not equal to each other throughout the flame brush, which is indicative of the anisotropic nature of the vorticity distribution within the flame brush.

In order to explain the differences in vorticity distribution within the flame brush due to the changes in $u^{\prime} / S_{L}$ and $g^{*}$, it is instructive to investigate the statistical behaviour of the terms of the vorticity and enstrophy transport equations (i.e., eqs. 2 and 4). The variations of the normalised values of $\overline{\left(t_{1 t} t_{1 t}\right)^{1 / 2}}$, $\overline{\left(t_{21 t} t_{21 t}\right)^{1 / 2}}, \overline{\left(t_{22 t} t_{22 t}\right)^{1 / 2}}, \overline{\left(t_{3 t} t_{3 t}\right)^{1 / 2}}$ and $\overline{\left(t_{4 t} t_{4 t}\right)^{1 / 2}}$ with $\tilde{c}$ are shown in Fig. 5 for all cases considered here where $\overline{\left(t_{q t} t_{q t}\right)^{1 / 2}}=\overline{\left[\left(\delta_{l J}-N_{l} N_{J}\right) t_{q l} t_{q J}\right]^{1 / 2}} \quad$ with $q=1,21,22,3,4$. The corresponding variations of the normalised values of $\overline{\left(t_{1 n} t_{1 n}\right)^{1 / 2}}, \overline{\left(t_{21 n} t_{21 n}\right)^{1 / 2}}, \overline{\left(t_{22 n} t_{22 n}\right)^{1 / 2}}$, $\overline{\left(t_{3 n} t_{3 n}\right)^{1 / 2}}$ and $\overline{\left(t_{4 n} t_{4 n}\right)^{1 / 2}}$ with $\tilde{c}$ are shown in Fig. 6 where $\overline{\left(t_{q n} t_{q n}\right)^{1 / 2}}=\overline{\left[\left(N_{l} N_{J}\right) t_{q l} t_{q J}\right]^{1 / 2}}$ with $q=1,21,22,3,4$. It is important to note that $\overline{\left(t_{q n} t_{q n}\right)^{1 / 2}}$ and $\overline{\left(t_{q t} t_{q t}\right)^{1 / 2}}$ (for $\left.q=1,21,22,3,4\right)$ are not the magnitudes of the transport equations of $\omega_{n}$ and $\omega_{t}$, respectively but these terms are to be interpreted as the magnitudes of the projections of the terms in the vorticity transport equation in local flame normal and tangential directions, respectively. 


$$
\begin{array}{cccc}
u_{(S e t-A)} S_{L}=3.0 & u^{\prime} / \underset{(S e t-B)}{S_{L}=5.0} & u^{\prime} \underset{(\text { Set }-C)}{S_{L}=7.5} & u^{\prime} \underset{(\text { Set }-D)}{S_{L}=10.0}
\end{array}
$$
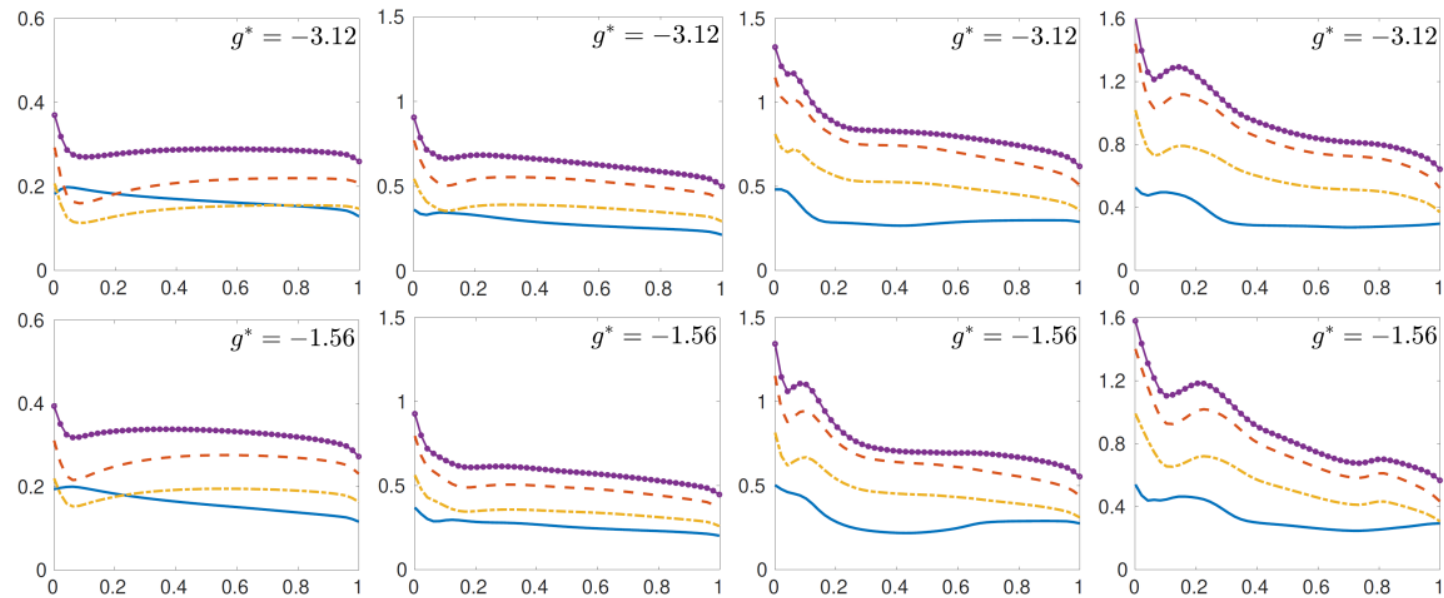

के
N
$\times$
है
हैं

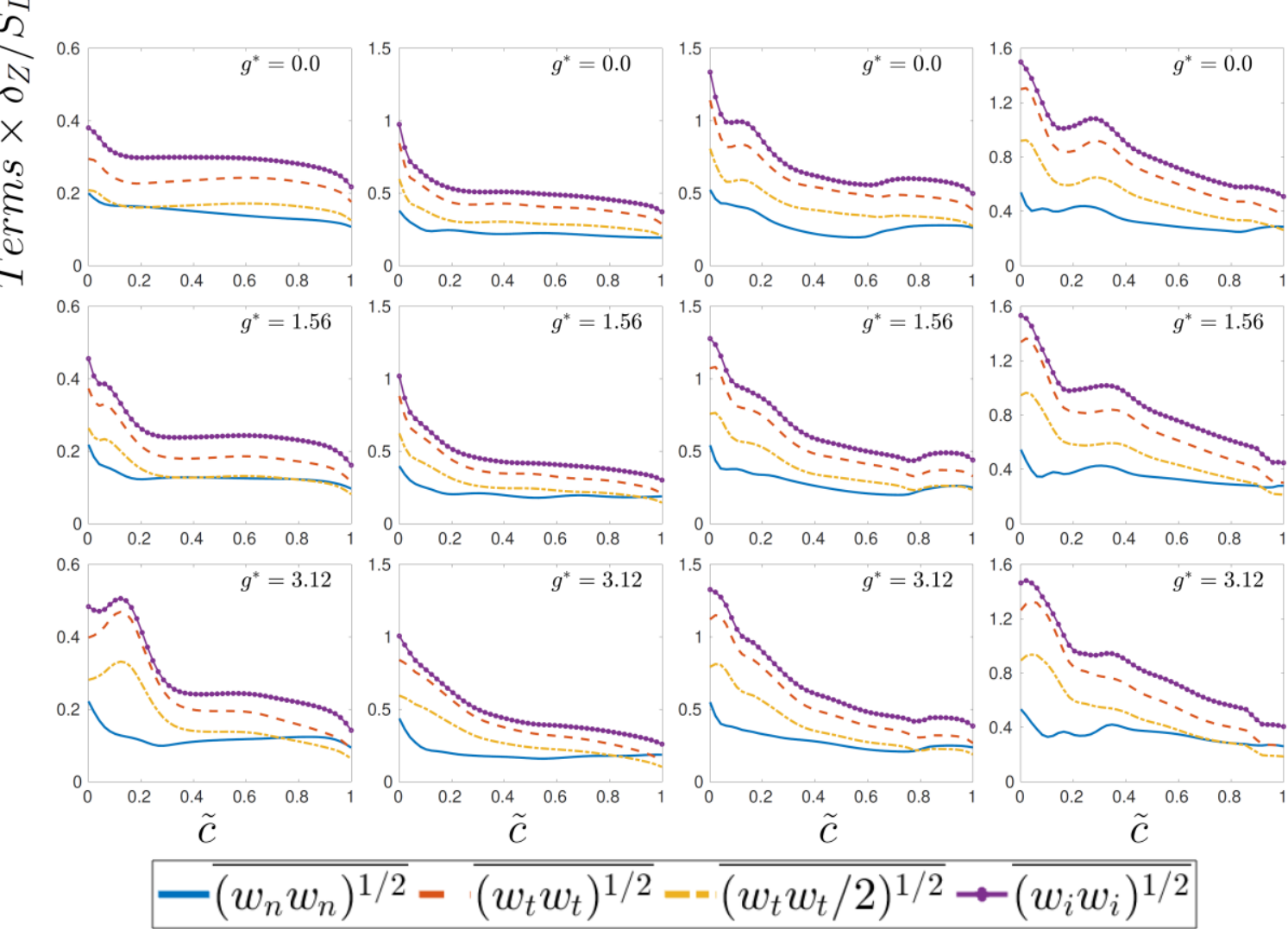

Figure 4: Variation of $\overline{\left(\omega_{l} \omega_{l}\right)^{1 / 2}} \times \delta_{Z} / S_{L}, \overline{\left(\omega_{n} \omega_{n}\right)^{1 / 2}} \times \delta_{Z} / S_{L}, \overline{\left(\omega_{t} \omega_{t}\right)^{1 / 2}} \times \delta_{Z} / S_{L}$ and $\left(\omega_{t} \omega_{t} / 2\right)^{1 / 2} \times \delta_{t h} / S_{L}$ with Favre averaged reaction progress variable $\tilde{c}$ for Set-A to Set-D $\left(1^{\text {st }}\right.$ $4^{\text {th }}$ column $)$ for $g^{*}=-3.12,-1.56,0.0,1.56$ and $3.12\left(1^{\text {st }}-5^{\text {th }}\right.$ row $)$. 
$\begin{array}{cccc}u^{\prime} \underset{(S e t-A)}{S_{L}=3.0} & u^{\prime} / \underset{(S e t-B)}{S_{L}=5.0} & u^{\prime} \underset{(\text { Set }-C)}{S_{L}=7.5} & u^{\prime} \underset{(\text { Set }-D)}{S_{L}=10.0}\end{array}$
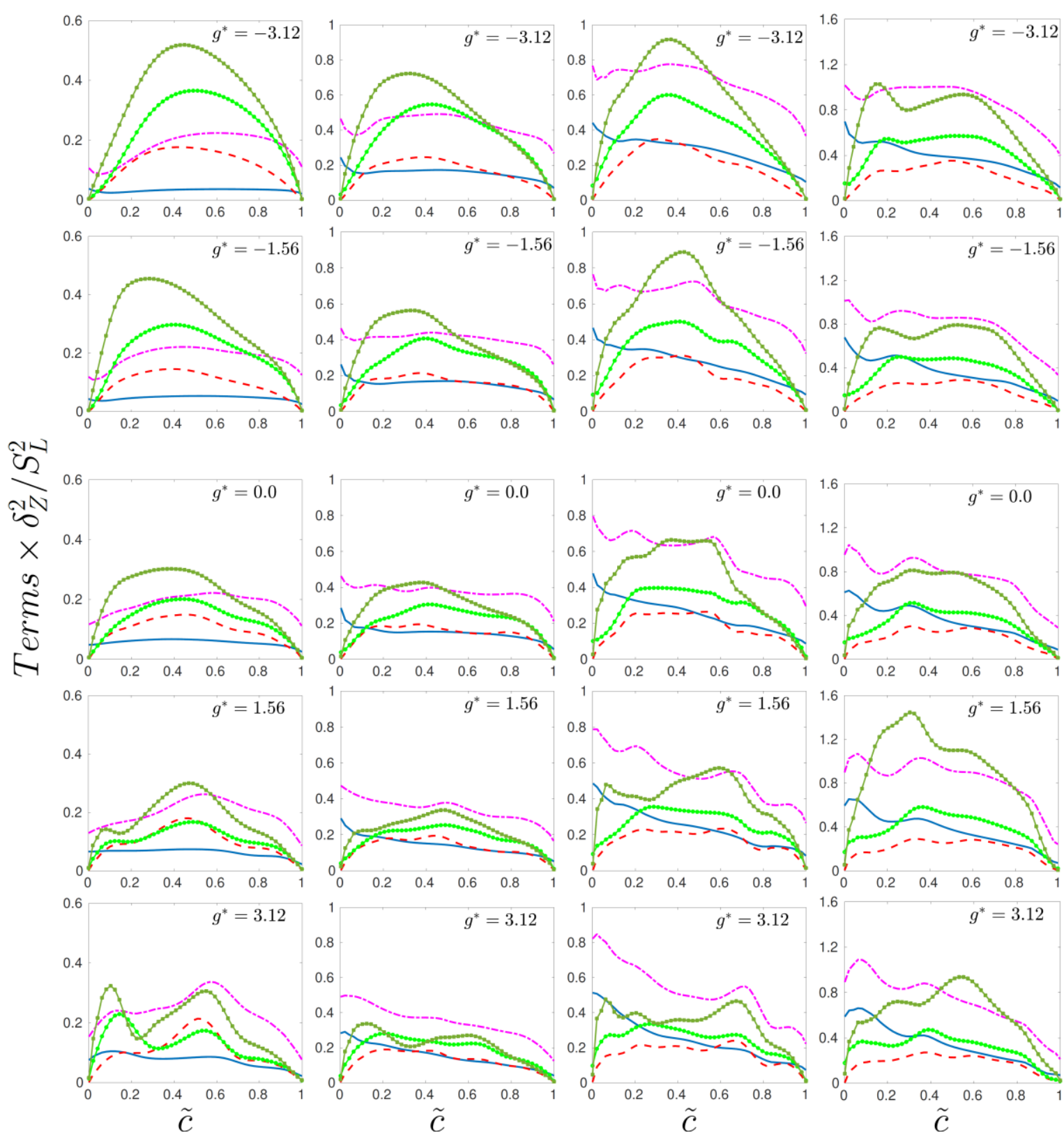

$-\overline{\left(t_{1 t} t_{1 t}\right)^{1 / 2}}-\overline{\left(t_{21 t} t_{21 t}\right)^{1 / 2}}-\overline{\left(t_{22 t} t_{22 t}\right)^{1 / 2}}=\overline{\left(t_{3 t} t_{3 t}\right)^{1 / 2}}=-\overline{\left(t_{4 t} t_{4 t}\right)^{1 / 2}}$

Figure 5: Variations of $\overline{\left(t_{1 t} t_{1 t}\right)^{1 / 2}} \times \delta_{Z}^{2} / S_{L}^{2}, \overline{\left(t_{21 t} t_{21 t}\right)^{1 / 2}} \times \delta_{Z}^{2} / S_{L}^{2}, \overline{\left(t_{22 t} t_{22 t}\right)^{1 / 2}} \times \delta_{Z}^{2} / S_{L}^{2}$, $\overline{\left(t_{3 t} t_{3 t}\right)^{1 / 2}} \times \delta_{Z}^{2} / S_{L}^{2}$ and $\overline{\left(t_{4 t} t_{4 t}\right)^{1 / 2}} \times \delta_{Z}^{2} / S_{L}^{2}$ with $\tilde{c}$ for Set-A to Set-D $\left(1^{\text {st }}-4^{\text {th }}\right.$ column $)$ for $g^{*}=$ $-3.12,-1.56,0.0,1.56$ and $3.12\left(1^{\text {st }}-5^{\text {th }}\right.$ row $)$. 


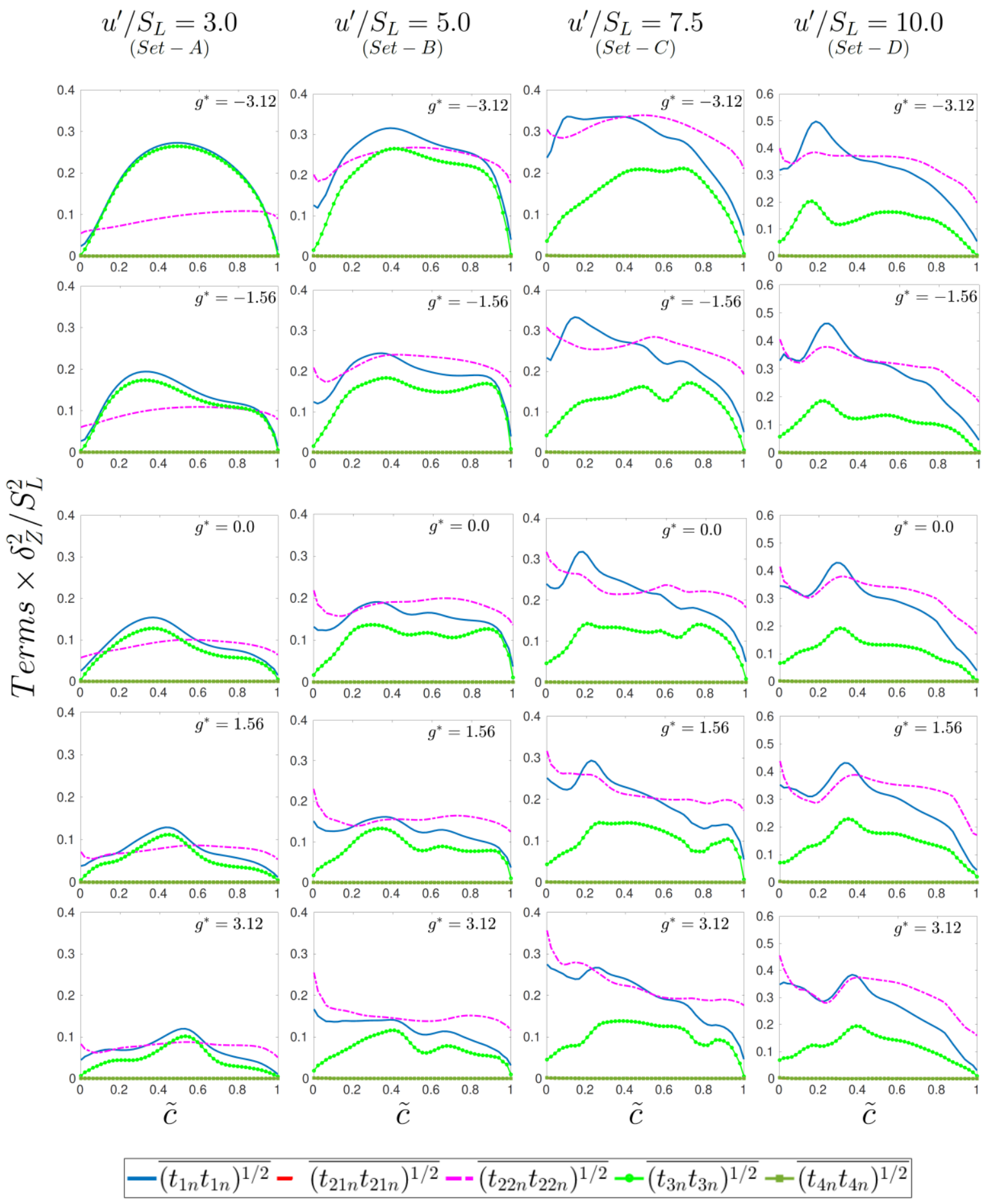

Figure 6: Variations of $\overline{\left(t_{1 n} t_{1 n}\right)^{1 / 2}} \times \delta_{Z}^{2} / S_{L}^{2}, \overline{\left(t_{21 n} t_{21 n}\right)^{1 / 2}} \times \delta_{Z}^{2} / S_{L}^{2}, \overline{\left(t_{22 n} t_{22 n}\right)^{1 / 2}} \times \delta_{Z}^{2} / S_{L}^{2}$, $\overline{\left(t_{3 n} t_{3 n}\right)^{1 / 2}} \times \delta_{Z}^{2} / S_{L}^{2}$ and $\overline{\left(t_{4 n} t_{4 n}\right)^{1 / 2}} \times \delta_{Z}^{2} / S_{L}^{2}$ with $\tilde{c}$ for Set-A to Set-D $\left(1^{\text {st }}-4^{\text {th }}\right.$ column $)$ for $g^{*}=$ $-3.12,-1.56,0.0,1.56$ and $3.12\left(1^{\text {st }}-5^{\text {th }}\right.$ row $)$.

A comparison between Figs. 5 and 6 indicates that the magnitude of the baroclinic torque contribution in the tangential direction (i.e., $\overline{\left(t_{4 t} t_{4 t}\right)^{1 / 2}}$ ) remains much greater than its vanishingly small magnitude in flame normal direction (i.e., $\overline{\left(t_{4 n} t_{4 n}\right)^{1 / 2}}$. For globally adiabatic unity Lewis number flames, the gas 
density $\rho$ can be expressed as: $\rho=\rho_{0} /(1+\tau c)^{21}$, which suggests that $\nabla \rho=-\rho_{0} \tau \nabla c /(1+\tau c)^{2}=$ $\rho^{2} \tau \vec{N}|\nabla c| / \rho_{0}$ and thus the baroclinic torque $(\nabla \rho \times \nabla p) / \rho^{2}$ does not have any component in the local flame normal direction. As the density gradient $\nabla \rho$ aligns collinearly with $\vec{N}$, there is no component of the term arising from the misalignment of density and viscous stress gradients in the local flame normal direction (i.e., $\overline{\left(t_{21 n} t_{21 n}\right)^{1 / 2}}=0.0$ ). It is important to note that the magnitude of the baroclinic torque contribution (i.e., $\overline{\left(t_{4 t} t_{4 t}\right)^{1 / 2}}$ ) remains significant for all cases and the magnitude of this term dominates over the magnitudes of other contributions of vorticity transport (i.e., $\overline{\left(t_{1 t} t_{1 t}\right)^{1 / 2}}, \overline{\left(t_{21 t} t_{21 t}\right)^{1 / 2}}$, $\overline{\left(t_{22 t} t_{22 t}\right)^{1 / 2}}$ and $\overline{\left(t_{3 t} t_{3 t}\right)^{1 / 2}}$ in the tangential direction for small and moderate values of $u^{\prime} / S_{L}$ (e.g., initial $u^{\prime} / S_{L}=3.0,5.0$ and 7.5 cases) for negative values of $g^{*}$ and this tendency is observed also for small turbulence intensities (e.g., initial $u^{\prime} / S_{L}=3.0$ case) without any body force (i.e., $g^{*}=0.0$ ). The magnitude of the contribution arising from the combined viscous diffusion and dissipation (i.e., $\overline{\left(t_{22 t} t_{22 t}\right)^{1 / 2}}$ and $\overline{\left(t_{22 n} t_{22 n}\right)^{1 / 2}}$ ) play leading order roles in both flame tangential and normal directions (see Figs. 5 and 6). The magnitude of the vortex-stretching term $\overline{\left(t_{1 t} t_{1 t}\right)^{1 / 2}}$ increases with increasing turbulence intensity $u^{\prime} / S_{L}$ irrespective of the values of $g^{*}$. Moreover, the relative importance of $\overline{\left(t_{1 t} t_{1 t}\right)^{1 / 2}}$ with respect to the leading order contributions of $\overline{\left(t_{22 t} t_{22 t}\right)^{1 / 2}}$ and $\overline{\left(t_{4 t} t_{4 t}\right)^{1 / 2}}$ increases with increasing $u^{\prime} / S_{L}$ and under these conditions $\overline{\left(t_{1 t} t_{1 t}\right)^{1 / 2}}$ and $\overline{\left(t_{22 t} t_{22 t}\right)^{1 / 2}}$ remain comparable for large values of $u^{\prime} / S_{L}$ similar to non-reacting flows. It can be seen from Fig. 6 that $\overline{\left(t_{1 n} t_{1 n}\right)^{1 / 2}}$ plays a leading order role for all turbulence intensities and $g^{*}$ values considered here, and $\overline{\left(t_{1 n} t_{1 n}\right)^{1 / 2}}$ remains comparable to $\overline{\left(t_{22 n} t_{22 n}\right)^{1 / 2}}$, which is similar to non-reacting flows for high turbulence intensities and/or positive values of $g^{*}$. The magnitude of the dilatation rate contribution $\overline{\left(t_{3 t} t_{3 t}\right)^{1 / 2}}$ plays a significant role in all cases. For negative values of $g^{*}$, the magnitude of $\overline{\left(t_{3 t} t_{3 t}\right)^{1 / 2}}$ remains comparable to the leading order contributions of $\overline{\left(t_{22 t} t_{22 t}\right)^{1 / 2}}$ and $\overline{\left(t_{4 t} t_{4 t}\right)^{1 / 2}}$ at small turbulence intensities (e.g., initial $u^{\prime} / S_{L}=3.0$ case) but the relative importance of the dilatation rate contribution $\overline{\left(t_{3 t} t_{3 t}\right)^{1 / 2}}$ diminishes with increasing $u^{\prime} / S_{L}$ and $g^{*}$. However, the magnitudes of $\overline{\left(t_{1 t} t_{1 t}\right)^{1 / 2}}$ and $\overline{\left(t_{3 t} t_{3 t}\right)^{1 / 2}}$ remain comparable for high positive values of $g^{*}$ and high turbulence intensities for the parameter range considered here. It can further be seen from Fig. 6 that the magnitudes of $\overline{\left(t_{3 n} t_{3 n}\right)^{1 / 2}}$ and $\overline{\left(t_{1 n} t_{1 n}\right)^{1 / 2}}$ 
remain comparable for small values of $u^{\prime} / S_{L}$ (e.g., initial $u^{\prime} / S_{L}=3.0$ case) but the relative importance of $\overline{\left(t_{3 n} t_{3 n}\right)^{1 / 2}}$ diminishes with increasing $u^{\prime} / S_{L}$ and $g^{*}$. Moreover, the magnitude of $\overline{\left(t_{3 n} t_{3 n}\right)^{1 / 2}}$ also decreases with increasing $g^{*}$. The magnitude of the term arising from the misalignment between the density gradient and viscous stress gradient in the flame tangential direction $\overline{\left(t_{21 t} t_{21 t}\right)^{1 / 2}}$ assumes nonnegligible values and remains comparable to $\overline{\left(t_{1 t} t_{1 t}\right)^{1 / 2}}$ for large turbulence intensities.

The vortex stretching term $\vec{t}_{1}$ can be expressed as ${ }^{10,14,15}$ :

$$
\vec{t}_{1}=\left(\omega_{i} \omega_{i}\right)^{1 / 2}\left[s_{\alpha} \cos \theta_{\alpha} \vec{e}_{\alpha}+s_{\beta} \cos \theta_{\beta} \vec{e}_{\beta}+s_{\gamma} \cos \theta_{\gamma} \vec{e}_{\gamma}\right]
$$

where $\theta_{\alpha}, \theta_{\beta}$ and $\theta_{\gamma}$ are the angles between $\vec{\omega}$ and $\vec{e}_{\alpha}, \vec{e}_{\beta}$, and $\vec{e}_{\gamma}$, respectively with $\vec{e}_{\alpha}, \vec{e}_{\beta}$, and $\vec{e}_{\gamma}$ being the eigenvectors corresponding to the most extensive, intermediate and the most compressive principal strain rates (i.e., $s_{\alpha}, s_{\beta}$ and $s_{\gamma}$ ), respectively. This leads to $t_{1 n}=\left(\omega_{i} \omega_{i}\right)^{1 / 2}\left[s_{\alpha} \cos \theta_{\alpha}\left(\vec{e}_{\alpha} \cdot \vec{N}\right)+\right.$ $\left.s_{\beta} \cos \theta_{\beta}\left(\vec{e}_{\beta} \cdot \vec{N}\right)+s_{\gamma} \cos \theta_{\gamma}\left(\vec{e}_{\gamma} \cdot \vec{N}\right)\right]$ and $t_{1 i} t_{1 i}=\left(\omega_{i} \omega_{i}\right)\left[s_{\alpha}^{2} \cos ^{2} \theta_{\alpha}+s_{\beta}^{2} \cos ^{2} \theta_{\beta}+s_{\gamma}^{2} \cos ^{2} \theta_{\gamma}\right]$. For small values of $u^{\prime} / S_{L}$ (e.g., initial $u^{\prime} / S_{L}=3.0$ case) $\vec{N}$ preferentially aligns collinearly with $\vec{e}_{\alpha}$ (i.e., $\left|\vec{e}_{\alpha} \cdot \vec{N}\right|=1.0,\left|\vec{e}_{\beta} \cdot \vec{N}\right| \approx 0.0$ and $\left.\left|\vec{e}_{\gamma} \cdot \vec{N}\right| \approx 0.0\right)$ where $s_{\alpha} \gg\left|s_{\beta}\right|,\left|s_{\gamma}\right|$ and $s_{\alpha} \approx \nabla \cdot \vec{u}=\left(s_{\alpha}+s_{\beta}+\right.$ $s_{\gamma}$ ) (not shown here but interested readers are referred to Refs. 10, 14 for the relevant statistics for turbulence parameters similar to Set-A and Set-B). Thus, $\overline{\left(t_{3 n} t_{3 n}\right)^{1 / 2}}=\left(\omega_{i} \omega_{i}\right)^{1 / 2} \mid(\nabla \cdot \vec{u})\left[\cos \theta_{\alpha}\left(\vec{e}_{\alpha}\right.\right.$. $\left.\vec{N})+\cos \theta_{\beta}\left(\vec{e}_{\beta} \cdot \vec{N}\right)+\cos \theta_{\gamma}\left(\vec{e}_{\gamma} \cdot \vec{N}\right)\right] \mid$ turns out to be $\overline{\left(t_{3 n} t_{3 n}\right)^{1 / 2}} \approx\left(\omega_{i} \omega_{i}\right)^{1 / 2}\left|s_{\alpha} \cos \theta_{\alpha}\left(\vec{e}_{\alpha} \cdot \vec{N}\right)\right| \approx$ $\overline{\left(t_{1 n} t_{1 n}\right)^{1 / 2}}$ for small turbulence intensities (e.g., initial $u^{\prime} / S_{L}=3.0$ and 5.0 cases) with this trend weakening with increasing $u^{\prime} / S_{L}$. Moreover, for small turbulence intensities (e.g., initial $u^{\prime} / S_{L}=3.0$ case), $t_{1 n} t_{1 n} \approx\left(\omega_{i} \omega_{i}\right) s_{\alpha}^{2} \cos \theta_{\alpha}$, which approaches $t_{1 i} t_{1 i} \approx\left(\omega_{i} \omega_{i}\right) s_{\alpha}^{2} \cos ^{2} \theta_{\alpha}$ because of $\left|\vec{e}_{\alpha} \cdot \vec{N}\right|=$ 1.0, $\left|\vec{e}_{\beta} \cdot \vec{N}\right| \approx 0.0$ and $\left|\vec{e}_{\gamma} \cdot \vec{N}\right| \approx 0.0$ along with $s_{\alpha} \gg\left|s_{\beta}\right|,\left|s_{\gamma}\right|$ and $s_{\alpha} \approx \nabla \cdot \vec{u}=\left(s_{\alpha}+s_{\beta}+s_{\gamma}\right)$. Therefore, the magnitudes of $\overline{\left(t_{1 t} t_{1 t}\right)^{1 / 2}}=\overline{\left(t_{1 l} t_{1 l}-t_{1 n} t_{1 n}\right)^{1 / 2}}$ remain small for small turbulence intensities (e.g., initial $u^{\prime} / S_{L}=3.0$ case), as can be observed from Fig. 5 .

It is worth noting that the terms $\vec{t}_{21}, \vec{t}_{3}$ and $\vec{t}_{4}$ arise due to thermal expansion as a result of heat release and thus the magnitudes of these terms in both flame normal and tangential directions assume 
vanishingly small values both on the unburned and the burned gas side of the flame brush. It can be seen from eq. 2 that the dilatation contribution destroys all vorticity components because of the predominantly positive dilatation rate $\partial u_{i} / \partial x_{i}$ values in premixed flames. The variations of the mean value of normalised dilatation rate $\overline{\left(\partial u_{l} / \partial x_{l}\right)} \times \delta_{Z} / S_{L}$ with $\tilde{c}$ for different values of $g^{*}$ for each set of turbulence parameters in Table I are shown in Fig. 7. It can be seen from Fig. 7 that the magnitude of $\overline{\left(\partial u_{l} / \partial x_{l}\right)} \times \delta_{Z} / S_{L}$ increases significantly with increasing negative magnitudes of $g^{*}$ for small values of $u^{\prime} / S_{L}$ (e.g., Set-A with initial $u^{\prime} / S_{L}=3.0$ ). However, this behaviour weakens with increasing $u^{\prime} / S_{L}$ and thus there is no significant impact of $g^{*}$ for large values of $u^{\prime} / S_{L}$ (e.g., Set-C and Set-D with initial values of $u^{\prime} / S_{L}=7.5$ and 10.0) because the influence of the body force weakens for large values of turbulence intensity, as discussed earlier. Figures 2 and 3 suggest that the extent of flame wrinkling (quantified in terms of flame surface area) increases significantly with increasing $g^{*}$ for small values of $u^{\prime} / S_{L}$ (e.g., Set-A with initial $u^{\prime} / S_{L}=3.0$ ) and thus the flame brush thickens with an increase in $g^{*}$. This phenomenon for small turbulence intensities (e.g., Set-A with initial $u^{\prime} / S_{L}=3.0$ ) leads to a decrease in $\overline{\left(\partial u_{\imath} / \partial x_{\imath}\right)} \times \delta_{Z} / S_{L}$ with an increase in $g^{*}$. A comparison between Figs. 5-7 reveals that the magnitudes of $\overline{\left(t_{3 t} t_{3 t}\right)^{1 / 2}}$ and $\overline{\left(t_{3 n} t_{3 n}\right)^{1 / 2}}$ do not change in proportion to $\overline{\left(\partial u_{l} / \partial x_{l}\right)}$ because of the correlation between $\omega_{i}$ and $\partial u_{i} / \partial x_{i}$ within the flame.

A comparison between Figs. 4-6 reveals that $\overline{\left(t_{4 t} t_{4 t}\right)^{1 / 2}}$ is principally responsible for either augmenting or arresting the decay of $\overline{\left(\omega_{t} \omega_{t}\right)^{1 / 2}}$ within the flame brush. Moreover, a comparison between Figs. 46 reveals that the baroclinic torque contribution is responsible for the anisotropic behaviour of vorticity components within the flame brush. Thus, it is worthwhile to consider the variations of density and pressure gradients (i.e., $\nabla \rho$ and $\nabla p$ ) within the flame brush for the cases considered here. In this respect, it is also worth noting that the density gradient statistics also affect the behaviour of $\vec{t}_{21}$ within the flame brush.

The variations of $|\nabla \rho| \times \delta_{Z} / \rho_{0}$ with $\tilde{c}$ for the cases considered here are shown in Fig. 8, which exhibits, in consistence with the variations of $\overline{\left(\partial u_{l} / \partial x_{l}\right)} \times \delta_{Z} / S_{L}$, a decreasing trend with increasing $g^{*}$ for small 
values of $u^{\prime} / S_{L}$ (e.g., Set-A with initial $u^{\prime} / S_{L}=3.0$ ). However, this behaviour weakens with increasing $u^{\prime} / S_{L}$, and $g^{*}$ does not have any significant influence for large values of $u^{\prime} / S_{L}$ (e.g., Set-C and Set-D with initial values of $u^{\prime} / S_{L}=7.5$ and 10.0), as discussed earlier. For globally adiabatic unity Lewis number combustion under low Mach number, $\nabla \rho$ can be expressed as $\nabla \rho=\rho^{2} \tau \vec{N}|\nabla c| / \rho_{0}$ and thus it does not have any component in the flame tangential direction. Accordingly, $\vec{t}_{21}$ and $\vec{t}_{4}$ do not have any components in the flame normal direction and their contributions are felt only in the flame tangential direction (see Figs. 5 and 6).
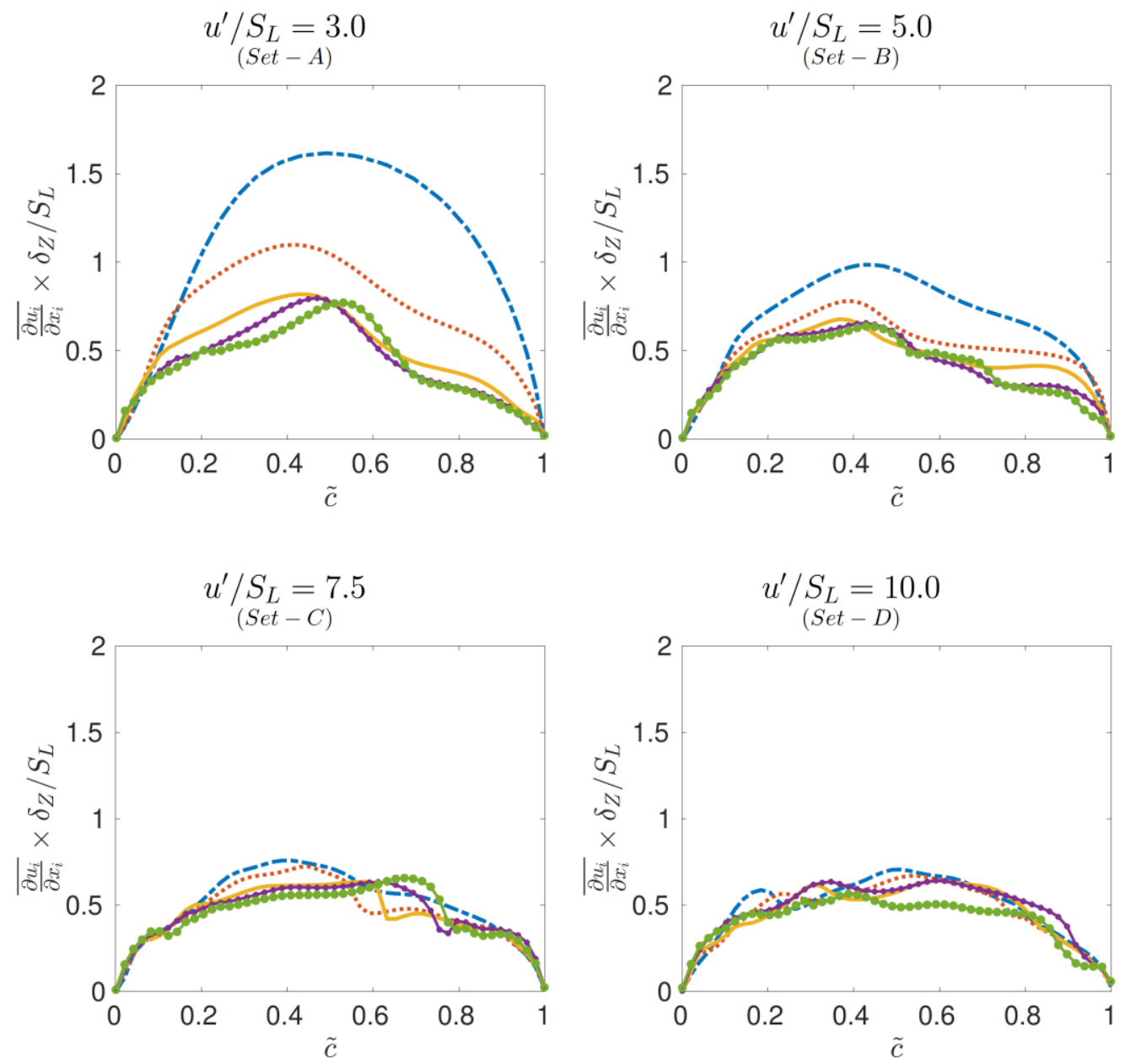

$$
--g^{*}=-3.12 \cdots g^{*}=-1.56-g^{*}=0.0 \multimap g^{*}=1.56 \rightarrow g^{*}=3.12
$$

Figure 7: Variations of $\overline{\left(\partial u_{\imath} / \partial x_{\imath}\right)} \times \delta_{Z} / S_{L}$ with $\tilde{c}$ for Set-A to Set-D for $g^{*}=$ $-3.12,-1.56,0.0,1.56$ and 3.12 . 

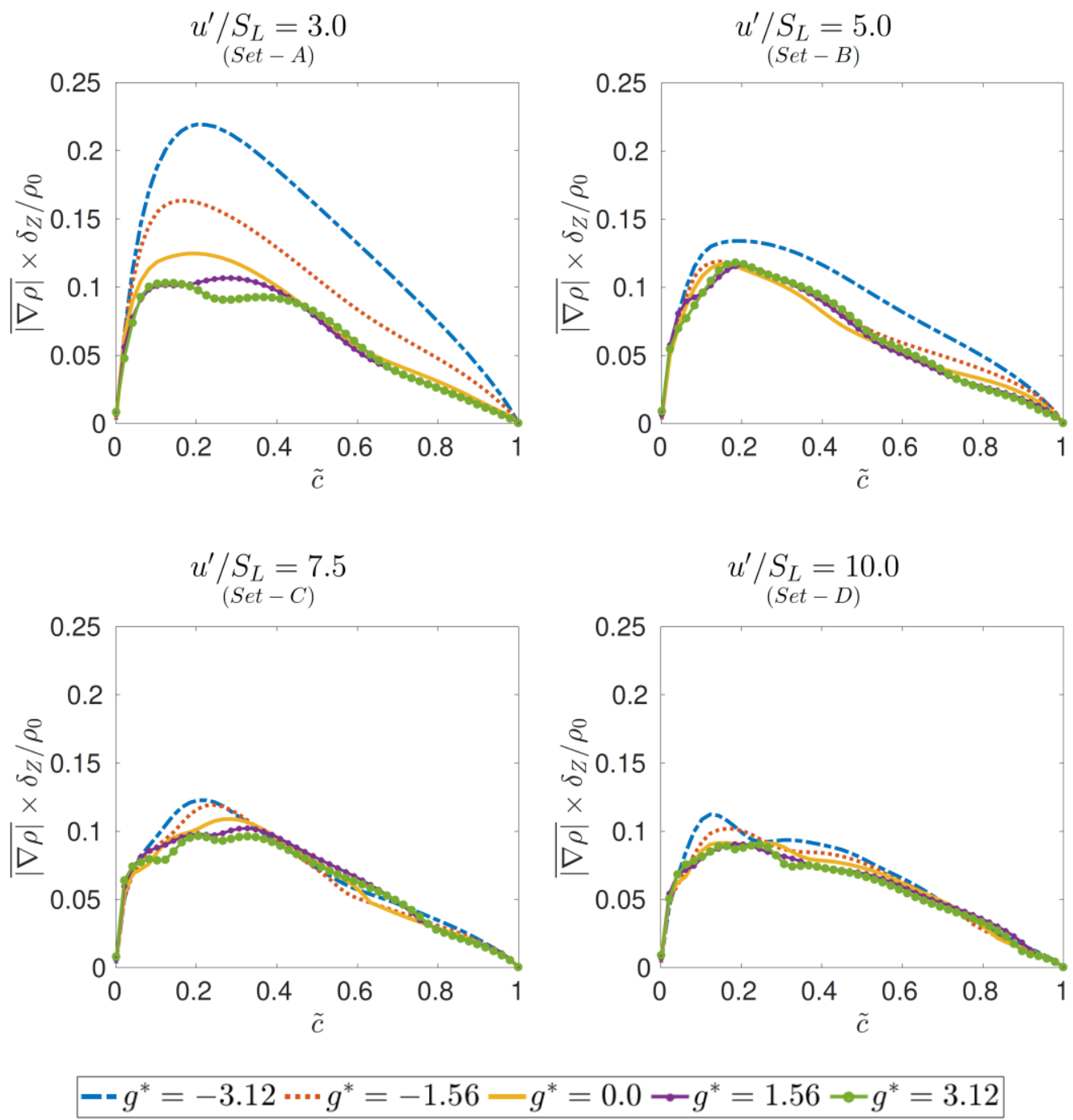

Figure 8: Variations of $\overline{|\nabla \rho|} \times \delta_{Z} / \rho_{0}$ with $\tilde{c}$ for Set-A to Set-D for $g^{*}=-3.12,-1.56,0.0,1.56$ and 3.12 .

The variations of $\left\{\overline{\left|(\nabla p)_{t}\right|}, \overline{\left|(\nabla p)_{n}\right|}\right.$ and $\left.\overline{|\nabla p|}\right\} \times \delta_{Z} / \rho_{0} S_{L}^{2}$ with $\tilde{c}$ for all cases considered here are reported in Fig. 9 where $\overline{\left|(\nabla p)_{n}\right|}$ and $\overline{\left|(\nabla p)_{t}\right|}$ are evaluated as $\overline{\left|(\nabla p)_{n}\right|}=\overline{|\vec{N} \cdot \nabla p|}$ and $\overline{\left|(\nabla p)_{t}\right|}=$

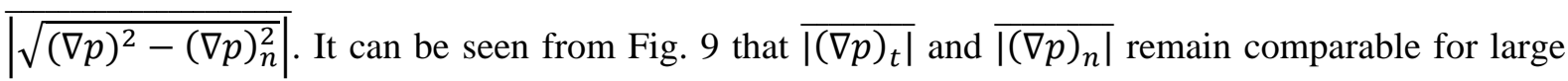
values of $u^{\prime} / S_{L}$ (e.g., Set-C and Set-D with initial values of $u^{\prime} / S_{L}=7.5$ and 10.0) irrespective of the value of $g^{*}$. However, for small values of $u^{\prime} / S_{L}$ (e.g., Set-A with initial values of $\left.u^{\prime} / S_{L}=3.0\right), \overline{\left|(\nabla p)_{n}\right|}$ dominates over $\overline{\left|(\nabla p)_{t}\right|}$ and this tendency decreases with an increase in the value of $g^{*}$. The flame normal pressure gradient magnitude $\overline{\left|(\nabla p)_{n}\right|}=\overline{|\vec{N} \cdot \nabla p|}$ is associated with locally normal flow acceleration, whereas the pressure gradient in tangential direction is induced by heat release in the 
surrounding flame wrinkles. The extent of flame wrinkling within the flame brush can be quantified by the departure of $\left|\vec{N} \cdot \vec{e}_{1}\right|$ from 1.0 where $\vec{e}_{1}$ is the unit vector in the direction of mean flame propagation. The variations of $\overline{\left|\vec{N} \cdot \vec{e}_{1}\right|}$ with $\tilde{c}$ for all cases are shown in Fig. 10 .

It can be seen from Figs. 9 and 10 that the cases with small values of $\overline{\left|\vec{N} \cdot \vec{e}_{1}\right|}$ exhibit comparable magnitudes of $\overline{\left|(\nabla p)_{t}\right|}$ and $\overline{\left|(\nabla p)_{n}\right|}$ (e.g., Set-C and Set-D for all values of $g^{*}$ and Set-B for high positive values of $g^{*}$ ), whereas $\overline{\left|(\nabla p)_{n}\right|}>\overline{\left|(\nabla p)_{t}\right|}$ is obtained for the cases which exhibit high values of $\overline{\left|\vec{N} \cdot \vec{e}_{1}\right|}$ (e.g., negative values of $g^{*}$ for Set-A). Figure 8 reveals that high magnitudes of $\overline{|\nabla \rho|}$ for decreasing negative values of $g^{*}$ for small turbulence intensities (e.g., Set-A and Set-B with initial $u^{\prime} / S_{L}=3.0$ and 5.0). This leads to increasingly strong contribution of baroclinic torque $(\nabla \rho \times \nabla p) / \rho^{2}$ in the flame tangential direction with decreasing $g^{*}$ in the cases for small turbulence intensities (e.g., Set-A and SetB with initial $u^{\prime} / S_{L}=3.0$ and 5.0), which exhibit comparable magnitudes of $\overline{\left|(\nabla p)_{t}\right|}$ for different values of $g^{*}$. The baroclinic torque may not only generate vorticity but can also locally damp vorticity depending on the angle between the vectors $\vec{\omega}$ and $\rho^{-2} \nabla \rho \times \nabla p$. This angle is characterized by $\theta_{p}=$ $\cos ^{-1}(\nabla \rho \times \nabla p) \cdot \vec{\omega} /(|\nabla \rho \times \nabla p||\vec{\omega}|)$. The variation of $\overline{\cos \theta_{p}}$ with $\tilde{c}$ for all cases considered here are shown in Fig. 11. It can be seen from Fig. 11 that the directions of $\vec{\omega}$ and $(\nabla \rho \times \nabla p) / \rho^{2}$ are completely independent of each other for leading and trailing edges of the flame brush. Within the flame brush, $\overline{\cos \theta_{p}}$ assumes relatively high magnitudes for negative values of $g^{*}$ when subjected to small levels of turbulence intensities (e.g., initial $u^{\prime} / S_{L}=3.0$ cases and weakly in the initial $u^{\prime} / S_{L}=5.0$ cases) where the tangential components of $(\nabla \rho \times \nabla p) / \rho^{2}$ contribute to the augmentation of $\overline{\left(\omega_{t} \omega_{t}\right)^{1 / 2}}$ within the flame brush. 


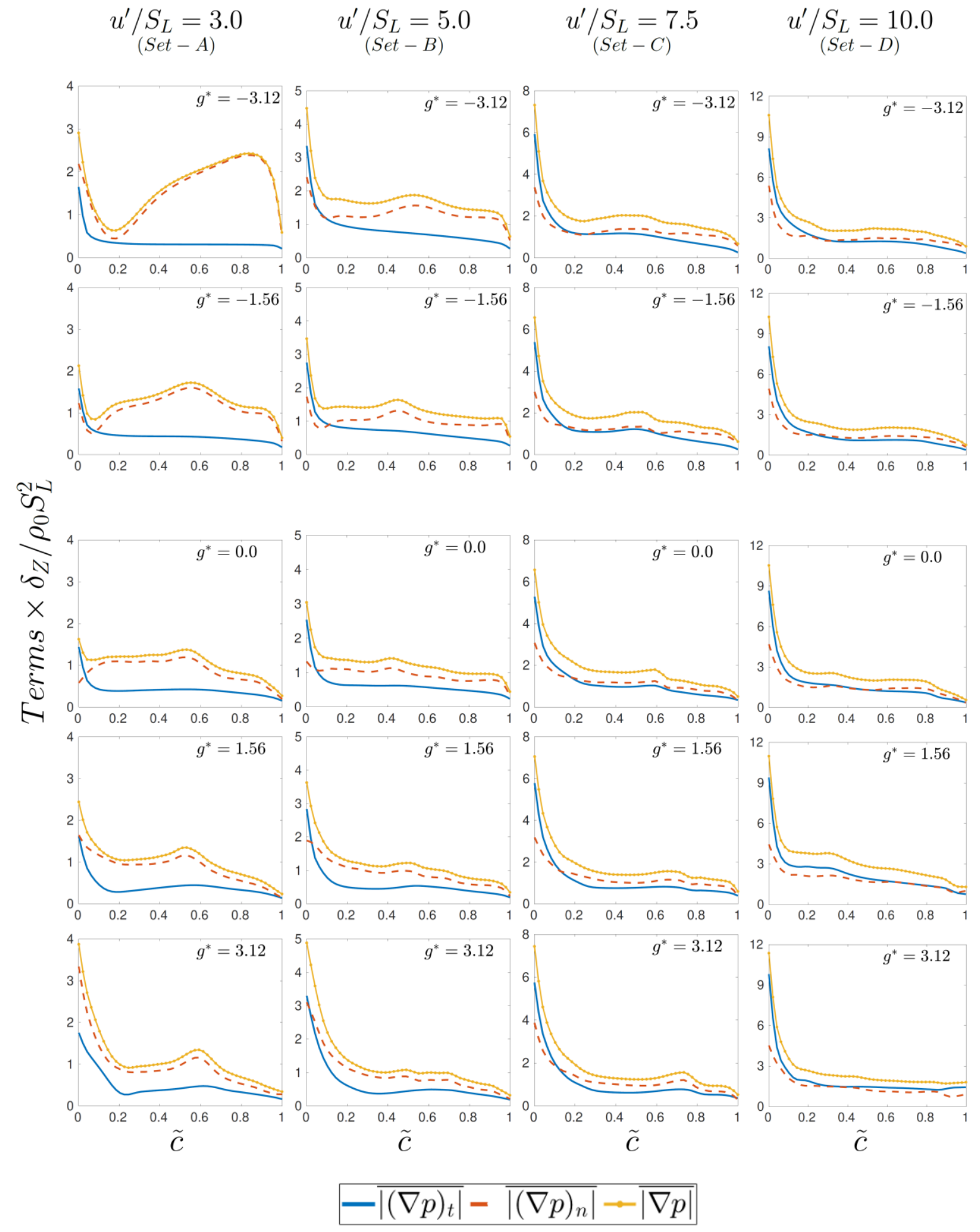

Figure 9: Variations of $\overline{\left|(\nabla p)_{t}\right|} \times \delta_{Z} / \rho_{0} s_{L}^{2}, \overline{\left|(\nabla p)_{n}\right|} \times \delta_{Z} / \rho_{0} S_{L}^{2}$ and $\overline{|\nabla p|} \times \delta_{Z} / \rho_{0} s_{L}^{2}$ with Favre averaged reaction progress variable $\tilde{c}$ for Set-A to Set-D $\left(1^{\text {st }}-4^{\text {th }}\right.$ column) for $g^{*}=$ $-3.12,-1.56,0.0,1.56$ and $3.12\left(1^{\text {st }}-5^{\text {th }}\right.$ row $)$. 
The variations of the normalised values of the terms of the right-hand side of the enstrophy transport equation (i.e., $T_{I}, T_{I I}, T_{I I I}, T_{I V}$ and $T_{V}$ ) with $\tilde{c}$ are shown in Fig. 12 for all cases considered here. It can be seen from Fig. 12 that the mean contribution of vortex-stretching term $T_{I}$ remains positive throughout the flame brush for all cases. The vortex-stretching term $T_{I}$ can be expressed as ${ }^{10,14,15}$ :

$$
T_{I}=\overline{2 \Omega\left(s_{\alpha} \cos ^{2} \theta_{\alpha}+s_{\beta} \cos ^{2} \theta_{\beta}+s_{\gamma} \cos ^{2} \theta_{\gamma}\right)}
$$

In all cases $\vec{\omega}$ exhibits preferential collinear alignment with the eigenvector $\vec{e}_{\beta}$ associated with the intermediate principal strain rate (not shown here) in accordance with several previous analyses for both reacting $^{9,10,14,34-36}$ and non-reacting flows ${ }^{26-33}$. As the intermediate principal strain rate $s_{\beta}$ is mostly positive, $T_{I}$ assumes positive values in all cases. However, the components of the vortex-stretching term $\vec{t}_{1}$ and the dilatation rate term $\vec{t}_{3}$ in the most extensive eigendirection cancel each other (see almost identical values of $\overline{\left(t_{1 n} t_{1 n}\right)^{1 / 2}}$ and $\overline{\left(t_{3 n} t_{3 n}\right)^{1 / 2}}$ in Fig. 6), and there is no component of $\vec{t}_{4}$ in the flame normal direction which is in collinear alignment with the most extensive strain rate eigendirection for initial $u^{\prime} / S_{L}=3.0$ cases for all values of $g^{*}$, and in $u^{\prime} / S_{L}=5.0$ cases for negative values of $g^{*}$. Therefore, in these cases $\vec{\omega}$ shows some alignment with the most compressive eigendirection along with the predominant alignment with the intermediate eigendirection, which suggests that negative $s_{\gamma} \cos ^{2} \theta_{\gamma}$ partially nullifies positive $s_{\beta} \cos ^{2} \theta_{\beta}$ to yield small positive values of $T_{I}$. 

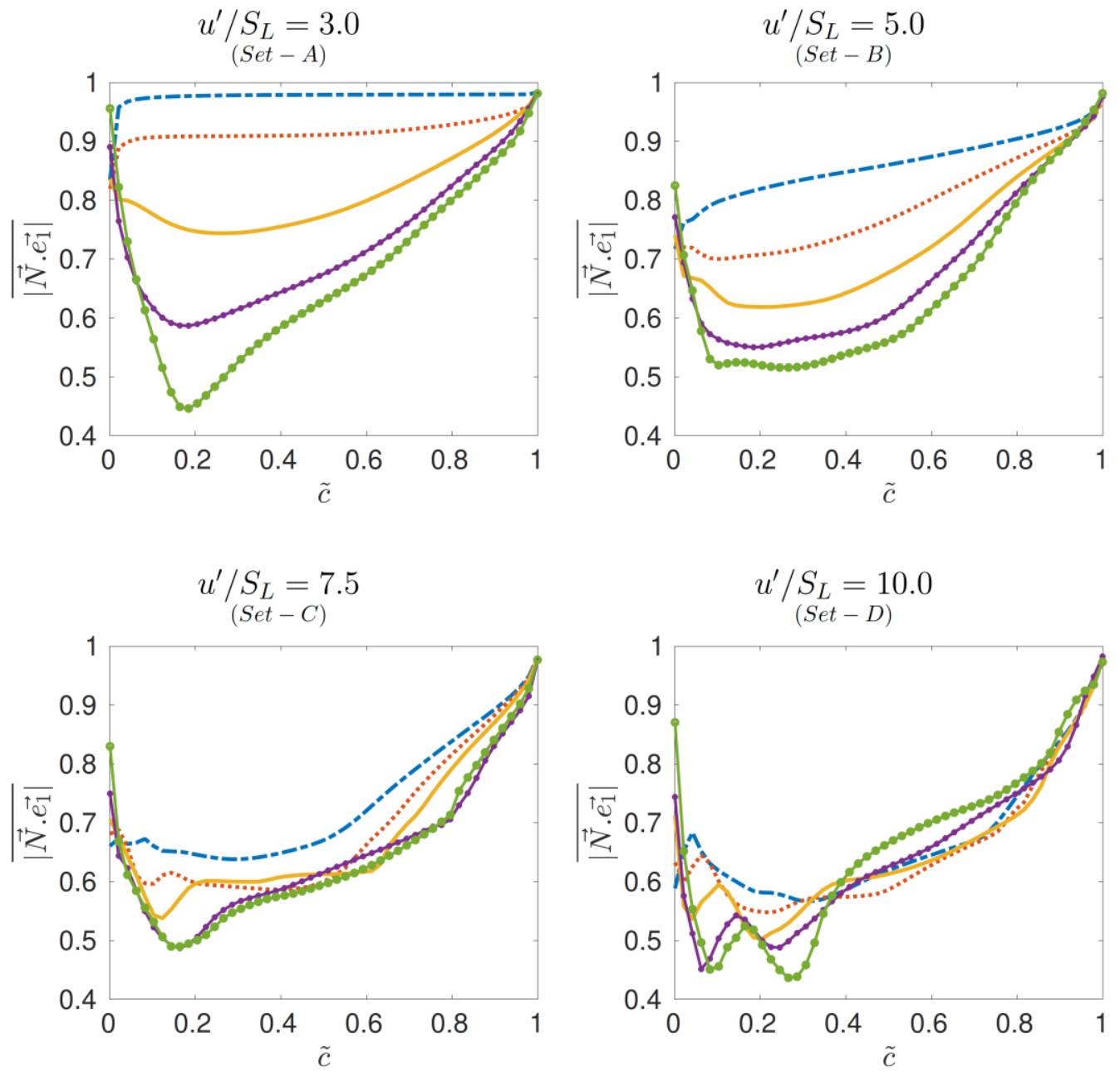

$$
--g^{*}=-3.12 \cdots g^{*}=-1.56-g^{*}=0.0 \multimap g^{*}=1.56 \multimap g^{*}=3.12
$$

Figure 10: Variations of $\overline{\left|\vec{N} \cdot \vec{e}_{1}\right|}$ with Favre averaged reaction progress variable $\tilde{c}$ for Set-A to Set-D for $g^{*}=-3.12,-1.56,0.0,1.56$ and 3.12. 

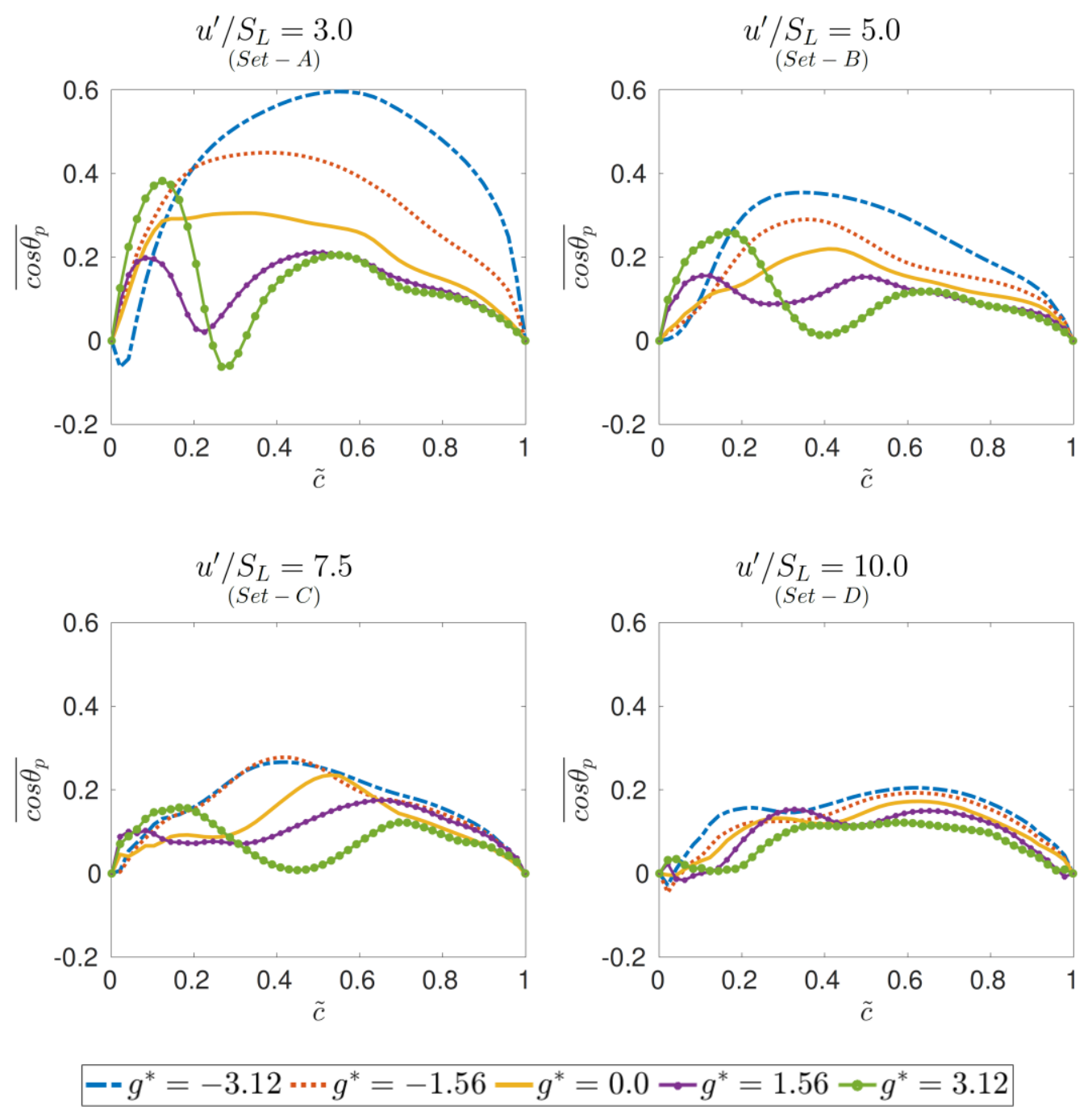

Figure 11: Variations of $\overline{\cos \theta_{p}}=\overline{(\nabla \rho \times \nabla p) \cdot \vec{\omega} /(|\nabla \rho \times \nabla p| \cdot|\vec{\omega}|)}$ with Favre averaged reaction progress variable $\tilde{c}$ for Set-A to Set-D for $g^{*}=-3.12,-1.56,0.0,1.56$ and 3.12.

The relative importance of source contribution of the vortex-stretching term $T_{I}$ strengthens with increasing values of both $u^{\prime} / S_{L}$ and $g^{*}$, and for large turbulence intensities $T_{I}$ becomes the most significant source term. For high values of $u^{\prime} / S_{L}$ and $g^{*}$, the leading order balance is maintained between $T_{I}$ and the combined molecular diffusion and viscous dissipation term $T_{I I I}$. The magnitude of the term arising from the misalignment of density gradient and viscous stress gradient remains negligible in comparison to the leading order sink contribution of the combined molecular diffusion and viscous dissipation term $T_{I I I}$. The term $T_{I I I}$ can be expressed as ${ }^{10,52}$ :

$$
T_{I I I}=\overline{(\mu / \rho) \nabla^{2} \Omega}+\overline{(\mu / 3 \rho) \vec{\omega} \cdot[\nabla \times \nabla(\nabla \cdot \vec{u})]}+f(\mu)-D_{v}
$$


where $f(\mu)$ represents the contributions from viscosity gradients and $-D_{v}=$ $-\overline{(\mu / \rho)\left(\partial \omega_{l} / \partial x_{l}\right)\left(\partial \omega_{l} / \partial x_{l}\right)}$ is the molecular dissipation of enstrophy. As dilatation rate $\nabla \cdot \vec{u}$ is a scalar the second term on right hand side of eq. 7 vanishes according to the mathematical identity $\nabla \times \nabla(\nabla \cdot \vec{u})=0$. For the present analysis, $\mu$ is taken to be constant so $f(\mu)$ is identically zero and the magnitude of $\overline{(\mu / \rho) \nabla^{2} \Omega}$ remains negligible in comparison to $\left(-D_{v}\right)=-\overline{(\mu / \rho)\left(\partial \omega_{l} / \partial x_{l}\right)\left(\partial \omega_{l} / \partial x_{l}\right)}$ and $T_{I I I}$ assumes negative values due to $\left(-D_{v}\right)$ for all cases considered here. The dilatation rate contribution $T_{I V}$ acts as a sink term for all cases but it is the most significant enstrophy sink for small values of $u^{\prime} / S_{L}$ (e.g., initial $u^{\prime} / S_{L}=3.0$ and 5.0 cases) and this trend strengthens (weakens) for increasing magnitudes of negative (positive) values of $g^{*}$. The relative strength of $T_{I V}$ diminishes with increasing $u^{\prime} / S_{L}$ but this term continues to play an important role even for initial $u^{\prime} / S_{L}=7.5$ and 10.0 cases. The baroclinic torque term $T_{V}$ acts as the most significant source term for flames with low and moderate turbulence intensities (e.g., initial $u^{\prime} / S_{L}=3.0$ and 5.0 cases) but its relative strength diminishes with increasing $g^{*}$, which is consistent with the observations made from Fig. 5. The relative importance of $T_{V}$ also weakens with increasing $u^{\prime} / S_{L}$, but even in initial $u^{\prime} / S_{L}=7.5$ and 10.0 cases, $T_{V}$ assumes values comparable to the magnitude of the vortex-stretching term $T_{I}$ and remains in approximate equilibrium with the dilatation rate contribution $T_{I I I}$. This suggests that although leading order contributions of $T_{I}$ and $T_{I I I}$ determine the mean enstrophy $\bar{\Omega}$ transport similar to non-reacting flows ${ }^{1-3,35}$ for high turbulence intensities especially for positive values of $g^{*}$, the dilatation rate and baroclinic torque terms (i.e., $T_{I V}$ and $T_{V}$ ), which arise due to density change and thermal expansion as a result of premixed combustion, continue to play important roles in the enstrophy evolution within the flame brush. A comparison between Figs. 2, 3 and 11 reveals that the baroclinic torque contribution is principally responsible for local instances of enstrophy generation and arresting the decay of enstrophy within the flame brush in the case of small turbulence intensities and this trend strengthens with the increasing magnitude of negative $g^{*}$.

For a given enstrophy isosurfaces, the kinematic equation is written as ${ }^{6-8,53,54}$ :

$$
\frac{\partial \Omega}{\partial t}+u_{j} \frac{\partial \Omega}{\partial x_{j}}=V_{\Omega}|\nabla \Omega|
$$


where $V_{\Omega}$ is the entrainment velocity ${ }^{6-8}$, which signifies that the iso-enstrophy surface moves with a propagation velocity of $\vec{u}+V_{\Omega} \vec{N}_{\Omega}$ where $\vec{N}_{\Omega}=-\nabla \Omega /|\nabla \Omega|$ is the normal vector of the iso-enstrophy surface. A comparison between eqs. 4 and 8 reveals that the net contribution of $\left(T_{I}+T_{I I}+T_{I I I}+T_{I V}+\right.$ $T_{V}$ ) provides the value of $\overline{V_{\Omega}|\nabla \Omega|}=\overline{\left(V_{\Omega}\right)_{s}} \overline{|\nabla \Omega|}$, where $\overline{|\nabla \Omega|}$ can be considered as the surface area to volume ratio of iso-enstrophy surface and $\overline{\left(V_{\Omega}\right)_{s}}$ is the area-weighted entrainment velocity value. It can be seen from Fig. 12 that $\left(T_{I}+T_{I I}+T_{I I I}+T_{I V}+T_{V}\right)$ predominantly assumes negative values, which implies that $\left.\overline{(V}_{\Omega}\right)_{S}$ is predominantly negative for the cases considered here, which can be verified from Fig. 13 where the variations of $\overline{\left(V_{\Omega}\right)_{S}} / S_{L}$ with $\tilde{c}$ are shown for Set-A to Set-D for different values of $g^{*}$. The predominance of negative values of entrainment velocity is consistent with the recent findings ${ }^{53,54}$ for the thin reaction zones regime flames. A turbulent/non-turbulent (T/NT) interface moves from the high vorticity region to a low vorticity region for a positive value of $V_{\Omega}$ and accordingly a negative value of $\overline{\left(V_{\Omega}\right)_{S}}$ implies that T/NT interfaces retreats into the high vorticity region. A comparison between Figs. 2 and 13 reveals that the movement of T/NT isosurfaces takes place from the burned gas to the unburned gas side of the flame. Figure 13 reveals that the order of magnitude $\left.\overline{(V}_{\Omega}\right)_{S}$ remains comparable to $S_{L}$, which is also consistent with the previous findings. ${ }^{53,54}$ Moreover, it can be seen from Fig. 13 that $g^{*}$ does not have any major influence on $\overline{\left(V_{\Omega}\right)}{ }_{S} / S_{L}$ for moderate and high turbulence intensities (e.g., Set-B to Set-D with initial $\left.u^{\prime} / S_{L}=5.0-10.0\right)$ but some minor influences can be discerned for low turbulence intensities (e.g., Set-A with initial $u^{\prime} / S_{L}=3.0$ ) where high positive values of $g^{*}$ (i.e., unstable stratification) tends to induce high negative values of $\overline{\left(V_{\Omega}\right)_{S}} / S_{L}$ but these effects remains prominent only towards the unburned gas side of the flame brush. 


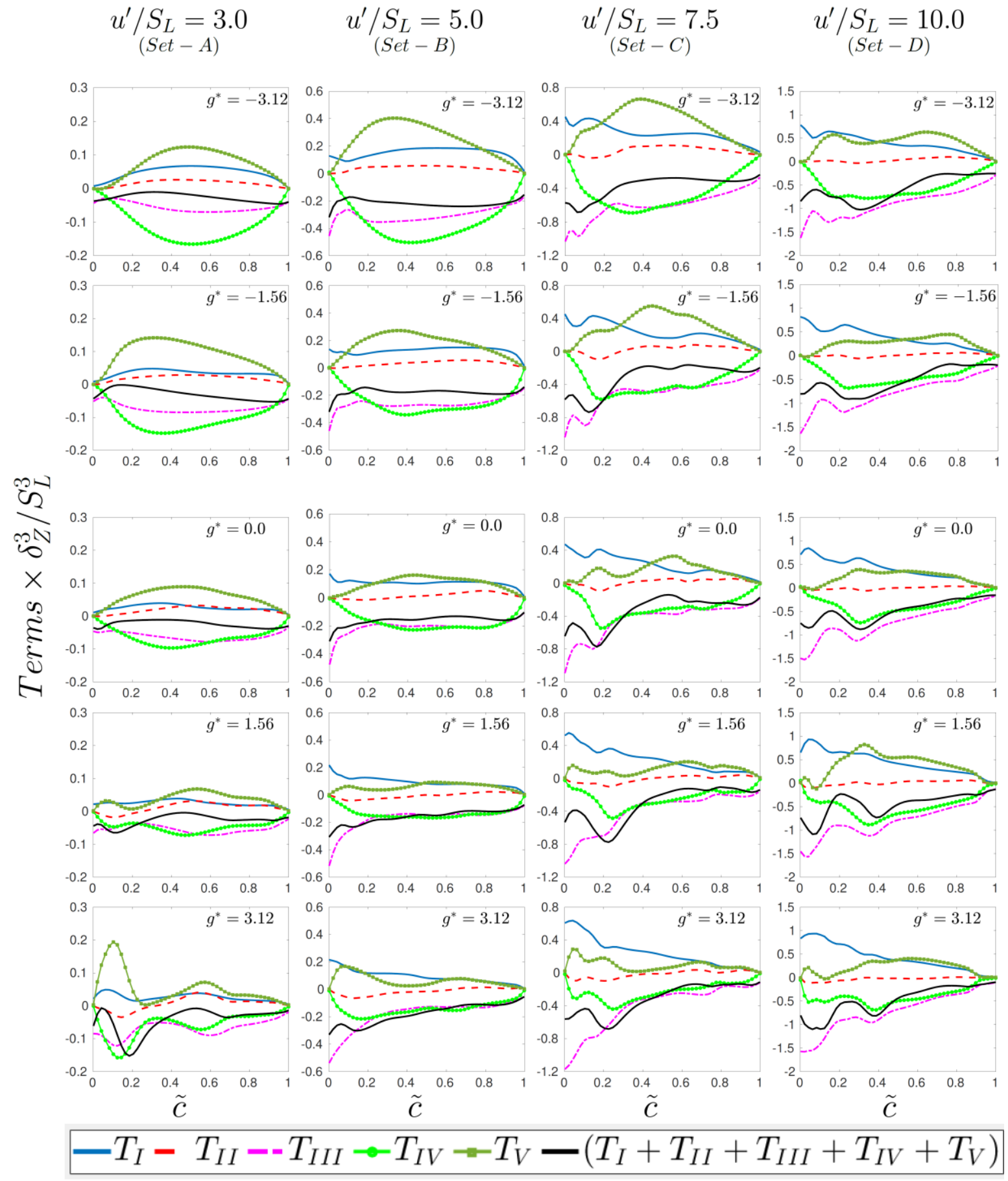

Figure 12: Variations of $T_{I} \times \delta_{Z}^{3} / S_{L}^{3}, T_{I I} \times \delta_{Z}^{3} / S_{L}^{3}, T_{I I} \times \delta_{Z}^{3} / S_{L}^{3}, T_{I V} \times \delta_{Z}^{3} / S_{L}^{3}, T_{V} \times \delta_{Z}^{3} / S_{L}^{3}$ and $\left(T_{I}+T_{I I}+T_{I I I}+T_{I V}+T_{V}\right) \times \delta_{Z}^{3} / S_{L}^{3}$ with $\tilde{c}$ for Set-A to Set-D $\left(1^{\text {st }}-4^{\text {th }}\right.$ column $)$ for $g^{*}=$ $-3.12,-1.56,0.0,1.56$ and $3.12\left(1^{\text {st }}-5^{\text {th }}\right.$ row $)$. 

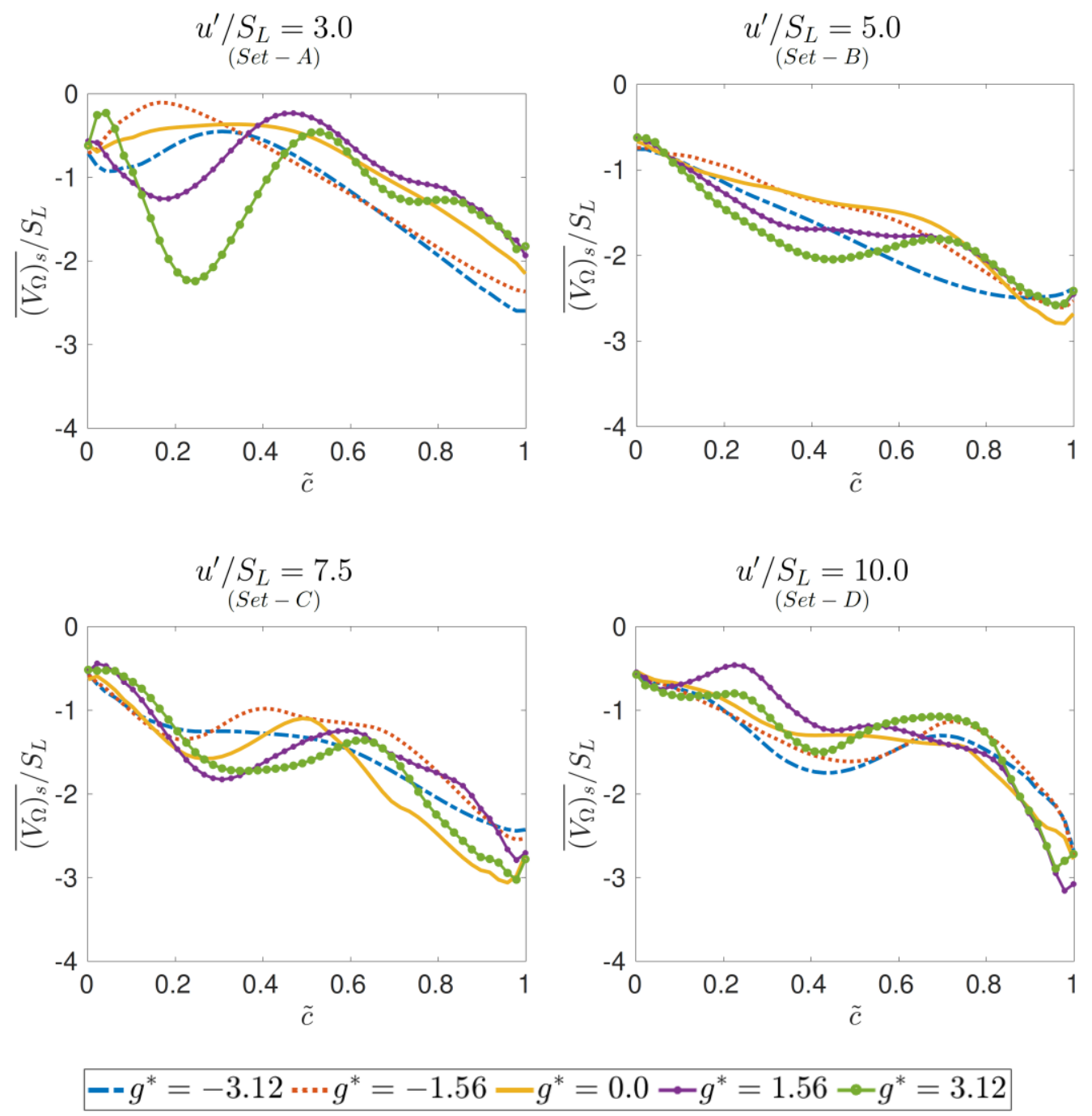

Figure 13: Variations of $\overline{\left(V_{\Omega}\right)_{s}} / S_{L}$ with $\tilde{c}$ for Set-A to Set-D for $g^{*}=-3.12,-1.56,0.0,1.56$ and 3.12.

\section{CONCLUSIONS}

The effects of body forces on the evolution of vorticity and enstrophy within the flame brush have been analysed using DNS data of freely propagating statistically planar turbulent premixed flames subjected to different turbulence intensities and Froude numbers. The cases investigated here are characterised by $K a>1$ and $D a \sim O(1)$ and are representative of the thin reaction zones regime of premixed turbulent combustion. ${ }^{37}$ It has been found that, under the conditions of the present study, enstrophy decreases significantly from the unburned to the burned gas side of the flame brush for high turbulence intensities and also for the strengthening of body force in the positive $x$-direction, which is representative of unstable stratification for the flames with the mean direction of flame propagation aligned with the 
negative $x$-direction. However, local instances of enstrophy augmentation within the flame brush has been observed for small turbulence intensities and this becomes increasingly prominent with the strengthening of body forces promoting stable stratification. The vorticity components have been shown to exhibit anisotropic behaviour within the flame brush. It has been demonstrated that the baroclinic torque term is principally responsible for this anisotropic behaviour. The vortex-stretching and viscous dissipation terms have been found to be the leading order source and sink terms respectively for large turbulence intensities but the source term due to baroclinic torque and the sink term due to dilatation continue to play significant roles for all cases within the parameter range considered here. However, the baroclinic torque and the sink term due to dilatation play increasingly important roles with decreasing turbulence intensities and this tendency strengthens further with the strengthening of body forces promoting stable stratification. The body force has been found not to have any major influence on the surface-weighted entrainment velocity. However, an unstable stratification for small turbulence intensities tends to promote high negative values of entrainment velocity only towards the unburned gas side of the flame brush. The surface weighted entrainment velocity assumes negative values throughout the flame brush in all cases and assumes values comparable to the laminar burning velocity which are consistent with previous findings. ${ }^{53,54}$

The qualitative nature of the findings of the current investigation is unlikely to be modified in the presence of detailed chemistry, as indicated in previous analyses. ${ }^{15,42}$ However, three-dimensional detailed chemistry DNS data for high values of turbulent Reynolds number will be necessary for deeper understanding of vorticity and enstrophy evolutions in premixed turbulent flames under the influence of external body forces. Furthermore, the present analysis does not address the wall-induced shear effects, which are likely to have significant influences on the vorticity transformation mechanisms for vorticity dynamics in turbulent reacting flows. ${ }^{52}$ Some of the issues will form the basis of future investigations in this regard. 


\section{DATA AVAILABILITY}

The data that support the findings of this study are available from the corresponding author upon reasonable request.

\section{ACKNOWLEDGEMENTS}

The authors are grateful to EPSRC (EP/P022286/1) for financial support. The computational support was provided by ARCHER (EP/K025163/1, EP/R029369/1), CIRRUS, and Rocket-HPC. 


\section{APPENDIX A}

The temporal evolutions of $c=0.3,0.5,0.7$ and 0.9 isosurfaces based on 3D views for with $g^{*}=$ $-3.12,0$ and $g^{*}=3.12$ are shown in Figs. 14 and 15 for Set-A and Set-D, respectively. The initial conditions for these isosurfaces correspond to flat square plates and thus are not explicitly shown here. It can be seen from Figs. 14 and 15 that the flame shape and wrinkling do not appreciably change between $2.25 t_{e}$ and $3.0 t_{e}$ (which corresponds to $75 \%$ and $100 \%$ of the simulation time, respectively). Therefore, a reasonable quasi-steady state has been obtained when the statistics were extracted.

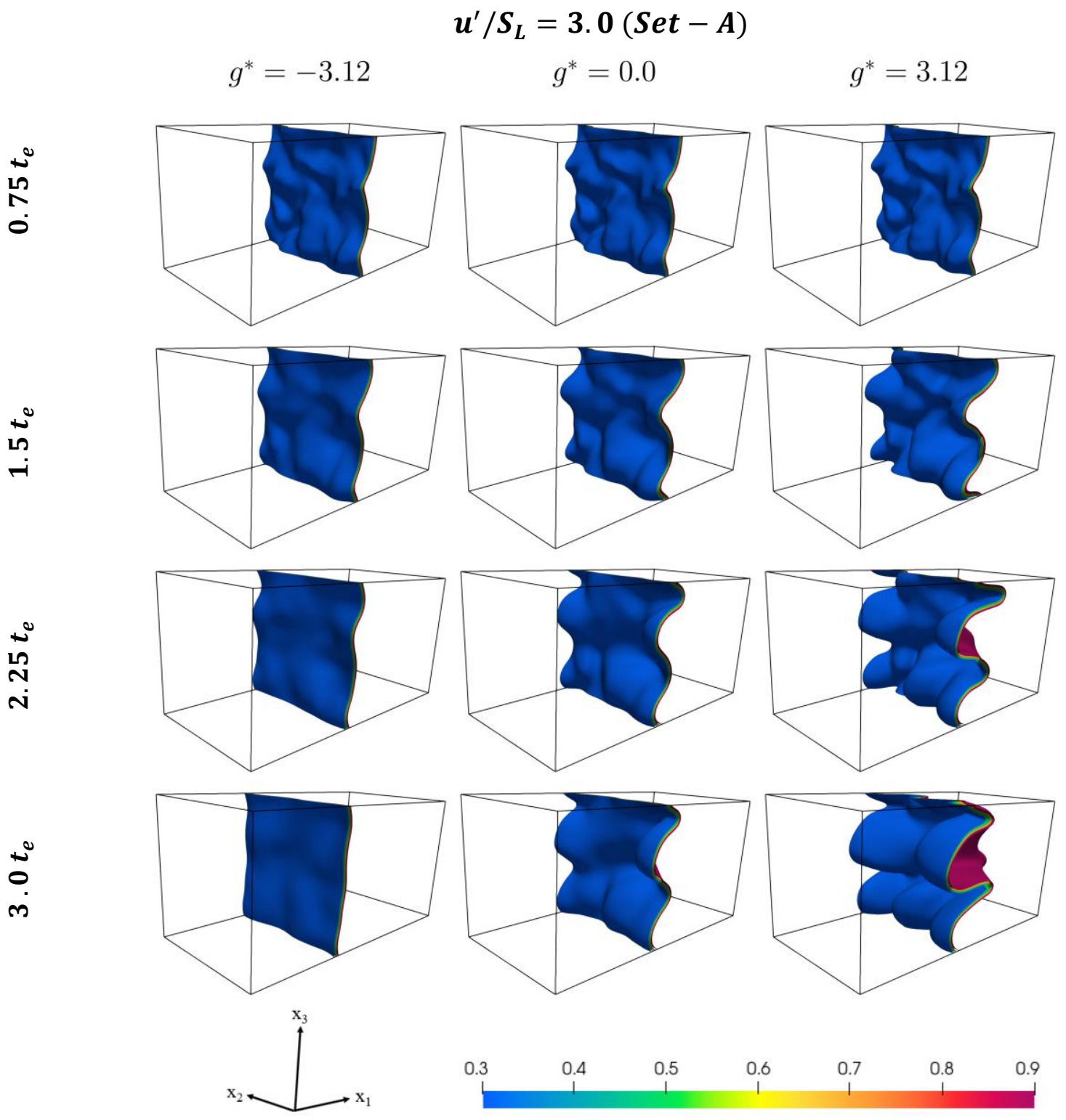

Figure 14: Temporal evolution of progress variable $c=0.3,0.5,0.7$ and 0.9 isosurfaces for Set-A with $g^{*}=-3.12,0.0$ and 3.12 


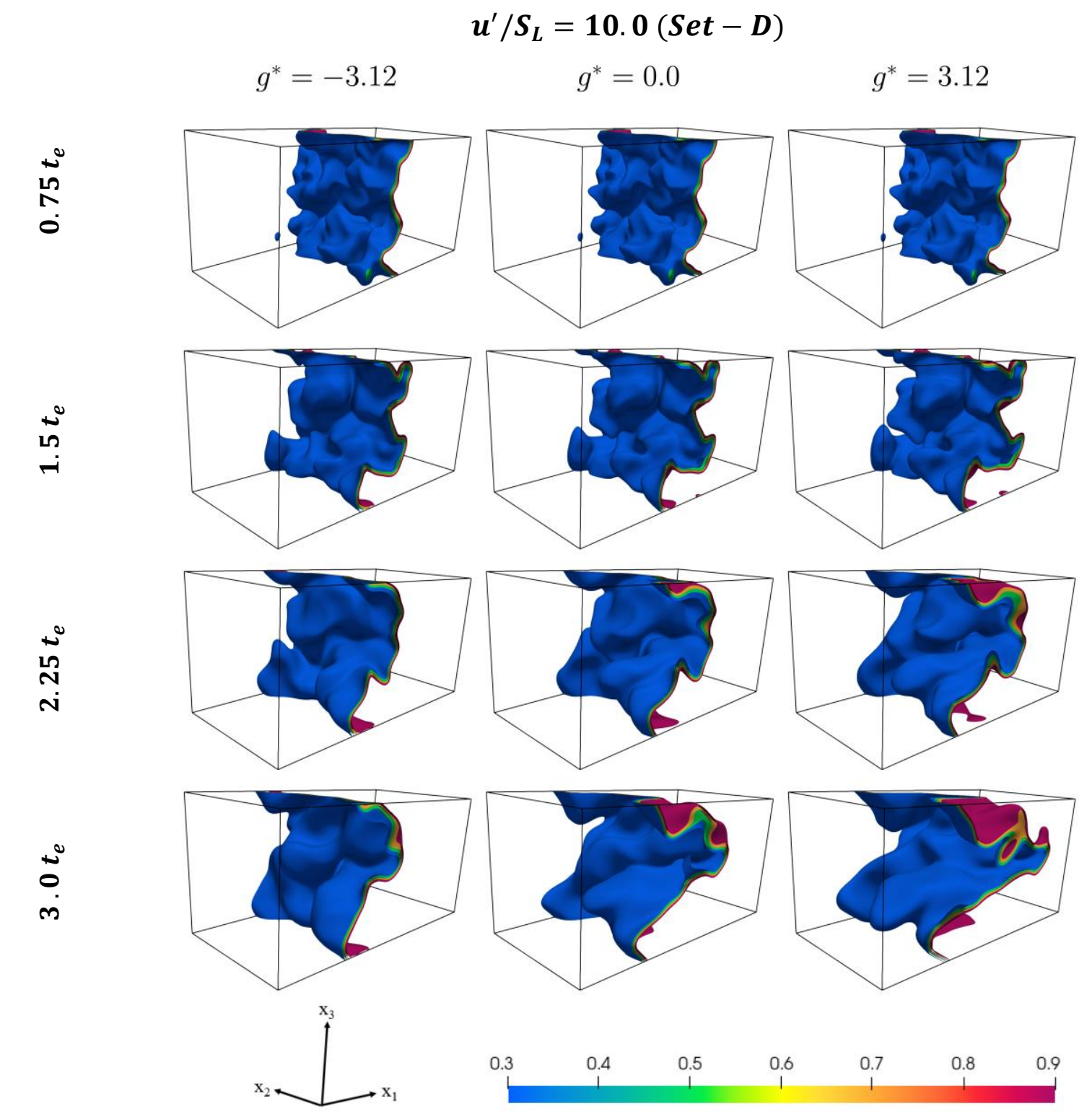

Figure 15: Temporal evolution of progress variable $c=0.3,0.5,0.7$ and 0.9 isosurfaces for Set-D with $g^{*}=-\mathbf{3 . 1 2}, 0.0$ and 3.12 


\section{REFERENCES}

${ }^{1}$ H. Tennekes, J.L. Lumley, A first course in turbulence, $1^{\text {st }}$ Edition, MIT Press, Cambridge, Massachusetts, USA, 1972.

${ }^{2}$ S.B. Pope, Turbulent Flows, $1{ }^{\text {st }}$ Edition, Cambridge University Press, Cambridge, UK, 2000.

${ }^{3}$ P.A. Durbin, B.A.P. Pettersson Reif, Statistical theory and modelling for turbulent flows, $2^{\text {nd }}$ Edition, Willey, 2010.

${ }^{4}$ A. Perry. M. Chong, A description of eddying motions and flow patterns using critical-point concepts. Ann. Rev. Fluid Mech. 19, 125-155, 1987.

${ }^{5}$ M. Chong, A. Perry, B. Cantwell, A general classification of three-dimensional flow fields, Phys. Fluids 2, 765-777, 1990.

${ }^{6} \mathrm{~S}$. Corrsin, Investigation of flow in an axially symmetrical heated jet of air, NACA Report No. W-94, 1943.

${ }^{7}$ P.A. Libby, On the prediction of intermittent turbulent flows, J. Fluid Mech., 68, 273-295, 1975.

${ }^{8}$ C. Dopazo, E.E. O’Brien Intermittency in Free Turbulent Shear Flows. In: Durst F., Launder B.E., Schmidt F.W., Whitelaw J.H. (eds) Turbulent Shear Flows I. Springer, Berlin, Heidelberg, 1979.

${ }^{9}$ P.E. Hamlington, A.Y. Poludnenko, E.S. Oran, Interactions between turbulence and flames in premixed reacting flows, Phys. Fluids, 23, 125111, 2011.

${ }^{10} \mathrm{~N}$. Chakraborty, Alignment of vorticity with strain rates in turbulent premixed flames, Eur. J. Mech. B-Fluids, 46, 201-220, 2014.

${ }^{11}$ A. L. Lipatnikov, S. Nishiki, T. Hasegawa, A direct numerical simulation study of vorticity transformation in weakly turbulent premixed flames. Phys. Fluids 26, 105104, 2014.

${ }^{12}$ N. Chakraborty, I. Konstantinou, A. Lipatnikov, Effects of Lewis number on vorticity and enstrophy transport in turbulent premixed flames, Phys. Fluids, 28,015109, 2016.

${ }^{13}$ C. Dopazo, L. Cifuentes, N. Chakraborty, Vorticity budgets in premixed combusting turbulent flows at different Lewis numbers, Phys. Fluids, 29, 045106, 2017.

${ }^{14}$ N. Chakraborty, L. Wang, I. Konstantinou, M. Klein, Vorticity statistics based on velocity and density-weighted velocity in premixed reactive turbulence, J. Turb., 18, 9, 2017. 
${ }^{15}$ V. Papapostolou, D.H. Wacks, M. Klein, N. Chakraborty, H. G. Im, Enstrophy transport conditional on local flow topologies in different regimes of premixed turbulent combustion, Scientific Reports, 7 , $11545,2017$.

${ }^{16}$ B. Karlovitz, D.W. Denniston, F.E. Wells, Investigation of turbulent flames, J. Chem. Phys., 19, $541,1951$.

${ }^{17}$ B. Karlovitz, D.W. Denniston Jr., D.H. Knapschaefer, F.E. Wells, Studies on turbulent flames, Proc. Combust. Inst., 4, 613-620,1953.

${ }^{18}$ K.N.C. Bray, P.A. Libby, Interaction effects in turbulent premixed flames, Phys. Fluids A, 19, 1687$1701,1976$.

${ }^{19}$ P. Moreau, A. Boutier, Laser velocimeter measurements in a turbulent flame, Proc. Combust. Inst., 16, 1747-1756, 1977.

${ }^{20}$ P.A. Libby, K.N.C. Bray, Counter gradient diffusion in premixed turbulent flames, AIAA J., 19, 205, 1981.

${ }^{21}$ K.N.C. Bray, P.A. Libby, J.B. Moss, Unified modelling approach for premixed turbulent combustion - Part I: General formulation, Combust. Flame, 61, 87-102, 1985.

${ }^{22}$ J.B. Moss, Simultaneous measurements of concentration and velocity in an open premixed flame, Combust. Sci. Technol., 22, 119-129, 1980.

${ }^{23}$ R. Borghi, D. Escudie, Assessment of a theoretical model of turbulent combustion by comparison with a simple experiment, Combust. Flame, 56, 149-164, 1984.

${ }^{24}$ J. Chomiak, J. Nisbet, Modelling variable density effects in turbulent flames, Combust. Flame, 102, 371-386, 1995.

${ }^{25}$ A.N. Lipatnikov, J. Chomiak, Effects of premixed flames on turbulence and turbulent scalar transport, Prog. Energy Combust. Sci., 36, 1-102, 2010.

${ }^{26}$ A. J. Majda, Vorticity, Turbulence, and Acoustics in Fluid Flow, SIAM Rev., 33 349-388,1991.

${ }^{27}$ J. Jimenez, Kinematic alignment effects in turbulent flows, Phys. Fluids A, 4, 652-654,1992.

${ }^{28}$ A. Tsinober, E. Kit, T. Dracos, Experimental investigation of the field of velocity gradients in turbulent flows, J. Fluid Mech., 242,169-192, 1992. 
${ }^{29}$ B.W. Zeff, D. Lanterman, D. R. McAllister, R. Roy, E.J. Kostelich, D.P. Lathrop, Measuring intense rotation and dissipation in turbulent flows, Nature, 421, 146-149,2003.

${ }^{30}$ B. Lüthi, A. Tsinober, W. Kinzelbach, Lagrangian measurement of vorticity dynamics in turbulent flow, J. Fluid Mech., 528, 87-118, 2005.

${ }^{31}$ P.E. Hamlington, J. Schumacher, W.J.A. Dahm, Local and nonlocal strain rate and vorticity alignment in turbulent flows, Phys. Rev. E., 77, 026303,2008.

${ }^{32}$ H. Xu, A. Pumir, E. Bodenschatz, The pirouette effect in turbulent flows, Nature Phys., 7, 709-712, 2011.

${ }^{33}$ K.K. Nomura, S.E. Elghobashi, The structure of inhomogeneous turbulence in variable density nonpremixed flames, Theor. Fluid Dyn., 5,153-175, 1993.

${ }^{34}$ O.N. Boratov, S.E. Elghobashi, R. Zhong, On the alignment of strain, vorticity and scalar gradient in turbulent, buoyant, nonpremixed flames, Phys. Fluids, 10, 2260-2268, 1996.

${ }^{35}$ F.A. Jaberi, D. Livescu, C.K. Madnia, Characteristics of chemically reacting compressible homogeneous turbulence, Phys. Fluids, 12, 1189-1210,2000.

${ }^{36}$ W. T. Ashurst, A. R. Kerstein, R. M. Kerr, C. H. Gibson, Alignment of vorticity and scalar gradient with strain rate in simulated Navier Stokes turbulence, Phys. Fluids 30, 2343, 1987.

${ }^{37}$ N. Peters, Turbulent combustion, 1st Edition, Cambridge University Press, 2000.

${ }^{38}$ A.N. Lipatnikov, V.A. Sabelnikov, S. Nishiki, T. Hasegawa: Does flame-generated vorticity increase turbulent burning velocity?, Phys. Fluids, 30, 081702, 2018.

${ }^{39}$ J. Chomiak and J. R. Nisbet, Modeling variable density effects in turbulent flames-Some basic considerations, Combust. Flame, 102, 371-386, 1995.

${ }^{40} \mathrm{D}$. Veynante and T. Poinsot, Effects of pressure gradients on turbulent premixed flames, J. Fluid Mech., 353, 83-114, 1997.

${ }^{41}$ K.W. Jenkins, R.S. Cant, Direct numerical simulation of turbulent flame kernels. In D. Knight \& L. Sakell, (eds) Recent Advances in DNS and LES: Proceedings of the Second AFOSR Conference, Rutgers - The State University of New Jersey, New Brunswick, USA, 191-202 (Kluwer, Dordrecht, 1999), 1999. 
${ }^{42}$ U. Ahmed, N.A.K Doan, J. Lai, M. Klein, N. Chakraborty, N. Swaminathan, Multiscale analysis of head-on quenching premixed turbulent flames, Phys. Fluids, 30,105102,2018.

${ }^{43}$ A.A. Wray, Minimal storage time advancement schemes for spectral methods, unpublished report, NASA Ames Research Center, California, 1990.

${ }^{44}$ T. Poinsot, S.K. Lele, Boundary conditions for direct simulation of compressible viscous flows, J. Comp. Phys., 101, 104-129, 1992.

${ }^{45}$ R. S. Rogallo, Numerical experiments in homogeneous turbulence, NASA Technicla Memorandum 81315, NASA AMES Research Centre, California, 1981.

${ }^{46}$ G. K. Batchelor and A. Townsend, Decay of turbulence in the final period, Proc. Roy. Soc. A, 194, 527-543, 1948.

${ }^{47}$ S. Zhang, C. J. Rutland, Premixed flame effects on turbulence and pressure-related terms, Combust. Flame, 102, 447-461, 1995.

${ }^{48}$ D. Veynante, A. Trouvé, K.N.C. Bray, T. Mantel, Gradient and counter-gradient scalar transport in turbulent premixed flames, J. Fluid Mech., 332, 263-293, 1997.

${ }^{49} \mathrm{~F}$. Charlette, C. Meneveau, D. Veynante, A power law wrinkling model for LES of premixed turbulent combustion, Part I: Non dynamic formulation and initial tests, Combust. Flame, 131, 159-181, 2002.

${ }^{50}$ I. Hun, K.Y. Huh, Roles of displacement speed on evolution of flame surface density for different turbulent intensities and Lewis numbers in turbulent premixed combustion, Combust. Flame, 152, 194$205,2008$.

${ }^{51}$ H. Reddy, J. Abraham, Two-dimensional Direct Numerical Simulation evaluation of the Flame Surface Density Model for flames developing from an ignition kernel in lean Methane/Air mixtures under engine conditions, Phys. Fluids, 24, 105108, 2012.

${ }^{52}$ J. Lai, N. Chakraborty, A. Lipatnikov, Vorticity and enstrophy transport in head-on quenching of turbulent premixed flames, Eur. J. Mech. Fluids/B, 65, 384-397, 2017.

${ }^{53}$ L. Cifuentes, A. M. Kempf, C. Dopazo, Local entrainment velocity in a premixed turbulent annular jet flame, Proc. Combust. Inst., 37, 2493-2501, 2019. 
${ }^{54}$ N. Chakraborty, M. Klein, H. G. Im, A comparison of entrainment velocity and displacement speed statistics in different regimes of turbulent premixed combustion, Proc. Combust. Inst., 38, https://doi.org/10.1016/j.proci.2020.06.241, 2020. 


\section{LIST OF FIGURES}

Figure 4: Schematic diagram of the simulation domain.

Figure 5: Distribution of $\left(\omega_{i} \omega_{i}\right)^{1 / 2} \times \delta_{Z} / S_{L}$ in the central $x_{1}-x_{2}$ plane at time when the statistics are extracted for Set-A to Set-D $\left(1^{\text {st }}-4^{\text {th }}\right.$ column $)$ for $g^{*}=-3.12,-1.56,0.0,1.56$ and $3.12\left(1^{\text {st }}-5^{\text {th }}\right.$ row). The contours of reaction progress variable $c=0.1$ to 0.9 (in steps of 0.1 from left to right) are also superimposed on the normalised vorticity magnitude distribution.

Figure 3: Variations of $A_{T} / A_{L}$ and $W_{T} / W_{L}$ with $g^{*}$ for all sets of turbulence parameters considered.

Figure 4: Variation of $\overline{\left(\omega_{l} \omega_{l}\right)^{1 / 2}} \times \delta_{Z} / S_{L}, \overline{\left(\omega_{n} \omega_{n}\right)^{1 / 2}} \times \delta_{Z} / S_{L}, \overline{\left(\omega_{t} \omega_{t}\right)^{1 / 2}} \times \delta_{Z} / S_{L} \quad$ and $\overline{\left(\omega_{t} \omega_{t} / 2\right)^{1 / 2}} \times \delta_{t h} / S_{L}$ with Favre averaged reaction progress variable $\tilde{c}$ for Set-A to Set$\mathrm{D}\left(1^{\text {st }}-4^{\text {th }}\right.$ column $)$ for $g^{*}=-3.12,-1.56,0.0,1.56$ and $3.12\left(1^{\text {st }}-5^{\text {th }}\right.$ row $)$.

Figure 5: Variations of $\overline{\left(t_{1 t} t_{1 t}\right)^{1 / 2}} \times \delta_{Z}^{2} / S_{L}^{2}, \overline{\left(t_{21 t} t_{21 t}\right)^{1 / 2}} \times \delta_{Z}^{2} / S_{L}^{2}, \overline{\left(t_{22 t} t_{22 t}\right)^{1 / 2}} \times \delta_{Z}^{2} / S_{L}^{2}$, $\overline{\left(t_{3 t} t_{3 t}\right)^{1 / 2}} \times \delta_{Z}^{2} / S_{L}^{2}$ and $\overline{\left(t_{4 t} t_{4 t}\right)^{1 / 2}} \times \delta_{Z}^{2} / S_{L}^{2}$ with $\tilde{c}$ for Set-A to Set-D $\left(1^{\text {st }}-4^{\text {th }}\right.$ column $)$ for $g^{*}=-3.12,-1.56,0.0,1.56$ and $3.12\left(1^{\text {st }}-5^{\text {th }}\right.$ row $)$.

Figure 6: Variations of $\overline{\left(t_{1 n} t_{1 n}\right)^{1 / 2}} \times \delta_{Z}^{2} / S_{L}^{2}, \overline{\left(t_{21 n} t_{21 n}\right)^{1 / 2}} \times \delta_{Z}^{2} / S_{L}^{2}, \overline{\left(t_{22 n} t_{22 n}\right)^{1 / 2}} \times \delta_{Z}^{2} / S_{L}^{2}$, $\overline{\left(t_{3 n} t_{3 n}\right)^{1 / 2}} \times \delta_{Z}^{2} / S_{L}^{2}$ and $\overline{\left(t_{4 n} t_{4 n}\right)^{1 / 2}} \times \delta_{Z}^{2} / S_{L}^{2}$ with $\tilde{c}$ for Set-A to Set-D $\left(1^{\text {st }}-4^{\text {th }}\right.$ column $)$ for $g^{*}=-3.12,-1.56,0.0,1.56$ and $3.12\left(1^{\text {st }}-5^{\text {th }}\right.$ row $)$.

Figure 7: Variations of $\overline{\left(\partial u_{l} / \partial x_{l}\right)} \times \delta_{Z} / S_{L}$ with $\tilde{c}$ for Set-A to Set-D for $g^{*}=$ $-3.12,-1.56,0.0,1.56$ and 3.12

Figure 8: Variations of $\overline{|\nabla \rho|} \times \delta_{Z} / \rho_{0}$ with $\tilde{c}$ for Set-A to Set-D for $g^{*}=-3.12,-1.56,0.0,1.56$ and 3.12 .

Figure 9: Variations of $\overline{\left|(\nabla p)_{t}\right|} \times \delta_{Z} / \rho_{0} S_{L}^{2}, \overline{\left|(\nabla p)_{n}\right|} \times \delta_{Z} / \rho_{0} S_{L}^{2}$ and $\overline{|\nabla p|} \times \delta_{Z} / \rho_{0} S_{L}^{2}$ with Favre averaged reaction progress variable $\tilde{c}$ for Set-A to Set-D $\left(1^{\text {st }}-4^{\text {th }}\right.$ column $)$ for $g^{*}=$ $-3.12,-1.56,0.0,1.56$ and $3.12\left(1^{\text {st }}-5^{\text {th }}\right.$ row $)$.

Figure 10: Variations of $\overline{\left|\vec{N} \cdot \vec{e}_{1}\right|}$ with Favre averaged reaction progress variable $\tilde{c}$ for Set-A to Set-D for $g^{*}=-3.12,-1.56,0.0,1.56$ and 3.12. 
Figure 11: Variations of $\overline{\cos \theta_{p}}=\overline{(\nabla \rho \times \nabla p) \cdot \vec{\omega} /(|\nabla \rho \times \nabla p| \cdot|\vec{\omega}|)}$ with Favre averaged reaction progress variable $\tilde{c}$ for Set-A to Set-D for $g^{*}=-3.12,-1.56,0.0,1.56$ and 3.12.

Figure 12: Variations of $T_{I} \times \delta_{Z}^{3} / S_{L}^{3}, T_{I I} \times \delta_{Z}^{3} / S_{L}^{3}, T_{I I I} \times \delta_{Z}^{3} / S_{L}^{3}, T_{I V} \times \delta_{Z}^{3} / S_{L}^{3}, T_{V} \times \delta_{Z}^{3} / S_{L}^{3} \quad$ and $\left(T_{I}+T_{I I}+T_{I I I}+T_{I V}+T_{V}\right) \times \delta_{Z}^{3} / S_{L}^{3}$ with $\tilde{c}$ for Set-A to Set-D $\left(1^{\text {st }}-4^{\text {th }}\right.$ column $)$ for $g^{*}=$ $-3.12,-1.56,0.0,1.56$ and $3.12\left(1^{\text {st }}-5^{\text {th }}\right.$ row $)$.

Figure 13: Variations of $\overline{\left(V_{\Omega}\right)}{ }_{S} / S_{L}$ with $\tilde{c}$ for Set-A to Set-D for $g^{*}=-3.12,-1.56,0.0,1.56$ and 3.12 .

Figure 14: Temporal evolution of progress variable $c=0.3,0.5,0.7$ and 0.9 isosurfaces for Set-A with $g^{*}=-3.12,0.0$ and 3.12.

Figure 15: Temporal evolution of progress variable $c=0.3,0.5,0.7$ and 0.9 isosurfaces for Set-D with $g^{*}=-3.12,0.0$ and 3.12 . 